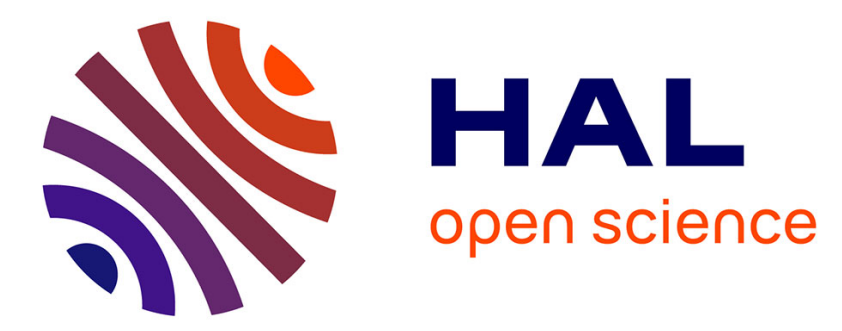

\title{
Battery State Estimation for a Single Particle Model With Electrolyte Dynamics
}

Scott J. Moura, Federico Bribiesca Argomedo, Reinhardt Klein, Anahita Mirtabatabaei, Miroslav Krstic

\section{- To cite this version:}

Scott J. Moura, Federico Bribiesca Argomedo, Reinhardt Klein, Anahita Mirtabatabaei, Miroslav Krstic. Battery State Estimation for a Single Particle Model With Electrolyte Dynamics. IEEE Transactions on Control Systems Technology, 2017, 25 (2), pp.453-468. 10.1109/TCST.2016.2571663 . hal-01478641

\section{HAL Id: hal-01478641 https://hal.science/hal-01478641}

Submitted on 2 Apr 2019

HAL is a multi-disciplinary open access archive for the deposit and dissemination of scientific research documents, whether they are published or not. The documents may come from teaching and research institutions in France or abroad, or from public or private research centers.
L'archive ouverte pluridisciplinaire HAL, est destinée au dépôt et à la diffusion de documents scientifiques de niveau recherche, publiés ou non, émanant des établissements d'enseignement et de recherche français ou étrangers, des laboratoires publics ou privés. 


\title{
Battery State Estimation for a Single Particle Model with Electrolyte Dynamics
}

\author{
Scott J. Moura, Federico Bribiesca Argomedo, Reinhardt Klein, Anahita Mirtabatabaei, Miroslav Krstic
}

\begin{abstract}
This paper studies a state estimation scheme for a reduced electrochemical battery model, using voltage and current measurements. Real-time electrochemical state information enables high-fidelity monitoring and high-performance operation in advanced battery management systems, for applications such as consumer electronics, electrified vehicles, and grid energy storage. This paper derives a single particle model with electrolyte (SPMe) that achieves higher predictive accuracy than the single particle model (SPM). Next, we propose an estimation scheme and prove estimation error system stability, assuming the total amount of lithium in the cell is known. The state estimation scheme exploits dynamical properties such as marginal stability, local invertibility, and conservation of lithium. Simulations demonstrate the algorithm's performance and limitations.
\end{abstract}

\section{INTRODUCTION}

This paper studies a state estimation algorithm based upon the single particle model with electrolyte dynamics - an electrochemical battery model. The algorithm features properties such as stability and conservation of lithium.

\section{A. Background \& Motivation}

Batteries are ubiquitous. They power a spectrum of devices, including consumer electronics, electrified vehicles, and smart grid systems. Control system technologies that enhance battery performance are of extreme interest. In particular, electrochemical model-based control systems provide visibility into operating regimes that induce degradation. This visibility enables a larger operational envelope to increase performance with respect to energy capacity, power capacity, and fast charge rates [1]. Electrochemical model-based state estimation is particularly challenging for several technical reasons. First, the dynamics are governed by a system of nonlinear partial differential algebraic equations [2], [3]. Second, local state observability does not hold globally [4]. Third, the model parameters vary widely with electrode chemistry, electrolyte, packaging, and time. Finally, cells in battery packs are generally

This work was supported in part by the National Science Foundation under Grant No. 1408107 and the Advanced Research Projects AgencyEnergy (ARPA-E), U.S. Department of Energy, under Award Number DEAR0000278.

S. Moura is with the Department of Civil and Environmental Engineering, University of California, Berkeley, CA 94720, USA (smoura@berkeley.edu)

F. Bribiesca Argomedo is with Université de Lyon - Laboratoire Ampère (CNRS UMR5005) - INSA de Lyon, 69621 Villeurbanne CEDEX, France (federico.bribiesca@insa-lyon.fr).

R. Klein and A. Mirtabatabaei are with the Robert Bosch LLC, Research and Technology Center, Palo Alto, CA 94304 USA (reinhardt.klein@us.bosch.com, Anahita.MirTabatabaei@us.bosch.com).

M. Krstic is with the Department of Mechanical and Aerospace Engineering, University of California, San Diego, La Jolla, CA 92093-0411, USA (krstic@ucsd.edu). heterogeneous with respect to their parameters, temperature, and state-of-charge. This motivates an intimate understanding of the mathematical model structure for observer design. In this paper, we address the first two technical challenges.

\section{B. Relevant Literature}

Over the past decade, the engineering literature on battery estimation has grown considerably rich with various algorithms, models, and applications. One may categorize this literature by the battery models each algorithm employs. Note, these studies all inherently assume the pack or modules can be conceptualized as an aggregate single cell. This is valid when cell balancing, binning, and temperature management maintain homogeneity across the pack or module.

The first category utilizes equivalent circuit models (ECMs). These models use circuit elements to mimic the input-output behavior of batteries [5]. The seminal paper by Plett [6] was one of the first to apply extended Kalman filtering (EKF) to ECMs for simultaneous state and parameter estimation. Over the past decade, a variety of ECM-based algorithms have been developed, including linear parameter varying observers [7], sliding mode observers [8], polynomial chaos [9], neural networks [10], unscented Kalman filters [11], adaptive Kalman filters [12], and particle filters [13].

The second category of literature considers electrochemical models, which account for the diffusion, intercalation, and electrochemical kinetics. Although these models can accurately predict internal state variables, their mathematical structure renders observer design challenging. Consequently, most approaches develop estimators for reduced-order models. The model reduction and observer design process are intimately intertwined, as simpler models ease estimation design at the expense of fidelity. Ideally, one seeks to derive a provably stable estimator for the highest fidelity electrochemical battery model possible. The first wave of studies utilize the "single particle model" (SPM) for estimator design [4], [14], [15], [16], [17]. The SPM idealizes each electrode as a single spherical porous particle by neglecting the electrolyte dynamics. This model is suitable for low C-rates, but loses fidelity at C-rates above C/2 (c.f. Section II-C). Recently, there has been progress on extending the SPM to include electrolyte dynamics [18], [19], [20], [21], [22]. In several cases, state observers have been designed via linearization and Luenberger observers [23] or EKFs [24]. State estimation designs have also emerged for other electrochemical models that incorporate electrolyte dynamics. Examples include spectral methods with output error injection [25], residue grouping with Kalman filtering 
[26], semi-separable structures with an EKF [27], discretetime realization algorithms with an EKF [28], and composite electrodes with nonlinear filters [29].

In all the aforementioned estimation studies for SPMs with electrolyte dynamics, a rigorous analysis of observer estimation error stability and conservation of lithium is lacking. Additionally, all these methods are reliant on a particular numerical discretization scheme. That is, they discretize the PDEs immediately, then apply analysis and estimation synthesis in the finite-dimensional domain. This paper performs analysis and estimation synthesis on the PDEs before discretization. The advantages are two-fold: (i) One does not have to revisit the estimator design if the discretization method is altered. (ii) The physical significance of the equations (and of the phenomena they represent) is retained regardless of the discretization method used at the implementation stage, thus yielding valuable insights as a by-product of the design process that are lost in other approaches. Unfortunately, it becomes increasingly difficult to prove estimation error stability as model complexity increases, as highlighted in the referenced literature. The core difficulty is lack of complete observability from voltage measurements.

\section{Main Contributions}

In this paper we advance the aforementioned research by designing a partial differential equation (PDE)-based observer for a SPMe. The observer design is unique, since it exploits fundamental electrochemistry dynamic properties, yielding a deeper insight in battery state estimation. We additionally prove stability of the estimation error system and conservation of lithium. Consequently, the article's main contributions are summarized as follows:

- Derivation of a single particle model with electrolyte (SPMe) oriented towards state estimation design. Similar models have been derived in [18], [19], [20], [21], [22]. We additionally analyze dynamical system properties that enable a provably convergent state estimator.

- A PDE-based state estimation design that exploits marginal stability, conservation of lithium, and output function invertibility to render convergent estimates. We also include a rigorous stability analysis of the estimation error systems.

\section{Outline}

The remainder of the paper is organized as follows. Section II derives the Single Particle Model with Electrolyte (SPMe) and analyzes relevant conservation properties, invertability, and accuracy relative to other electrochemical models. Section III derives the state estimation scheme for each constitutive subsystem. Section IV analyzes stability of the estimation error dynamics. The estimator is demonstrated via simulations in Section V. Key conclusions are provided in Section VI.

\section{Single Particle Model with Electrolyte}

\section{A. The Doyle Fuller Newman (DFN) Model}

In this section we describe the assumptions and steps followed to derive the SPMe model. First, we summarize

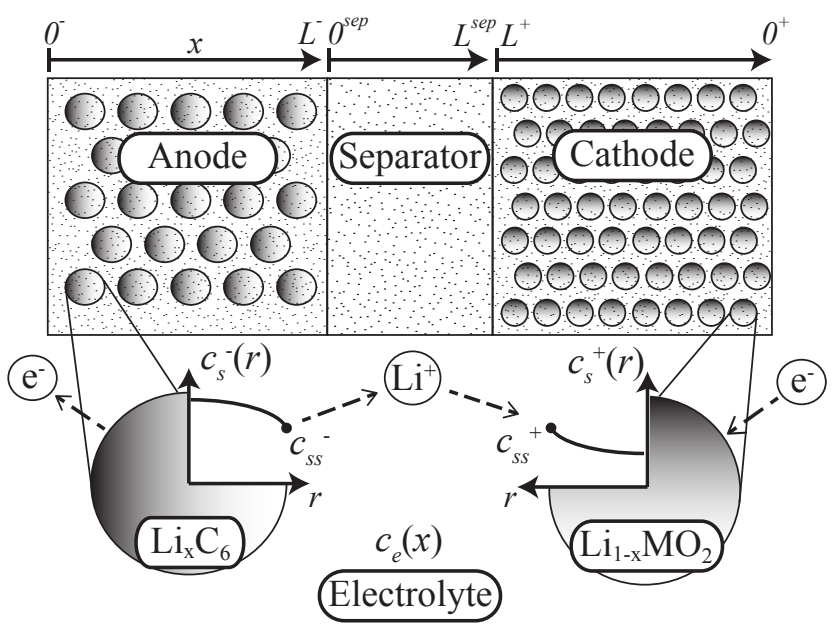

Fig. 1. Schematic of the Doyle-Fuller-Newman model [2]. The model considers two phases: the solid and electrolyte. In the solid, states evolve in the $x$ and $r$ dimensions. In the electrolyte, states evolve in the $x$ dimension only. The cell is divided into three regions: anode, separator, and cathode.

the DFN model in Fig. 1 to predict the evolution of lithium concentration in the solid $c_{s}^{ \pm}(x, r, t)$, lithium concentration in the electrolyte $c_{e}(x, t)$, solid electric potential $\phi_{s}^{ \pm}(x, t)$, electrolyte electric potential $\phi_{e}(x, t)$, ionic current $i_{e}^{ \pm}(x, t)$, and molar ion fluxes $j_{n}^{ \pm}(x, t)$. The governing equations are:

$$
\begin{aligned}
& \frac{\partial c_{s}^{ \pm}}{\partial t}(x, r, t)=\frac{1}{r^{2}} \frac{\partial}{\partial r}\left[D_{s}^{ \pm} r^{2} \frac{\partial c_{s}^{ \pm}}{\partial r}(x, r, t)\right], \\
& \varepsilon_{e}^{j} \frac{\partial c_{e}^{j}}{\partial t}(x, t)=\frac{\partial}{\partial x}\left[D_{e}^{\mathrm{eff}}\left(c_{e}^{j}\right) \frac{\partial c_{e}^{j}}{\partial x}(x, t)+\frac{1-t_{c}^{0}}{F} i_{e}^{j}(x, t)\right]
\end{aligned}
$$

for $j \in\{-$, sep, +$\}$ and

$$
\begin{aligned}
& \sigma^{\mathrm{eff}, \pm} \cdot \frac{\partial \phi_{s}^{ \pm}}{\partial x}(x, t)=i_{e}^{ \pm}(x, t)-I(t), \\
& \kappa^{\mathrm{eff}}\left(c_{e}\right) \cdot \frac{\partial \phi_{e}}{\partial x}(x, t)=-i_{e}^{ \pm}(x, t)+\kappa^{\mathrm{eff}}\left(c_{e}\right) \cdot \frac{2 R T}{F}\left(1-t_{c}^{0}\right) \\
& \times\left(1+\frac{d \ln f_{c / a}}{d \ln c_{e}}(x, t)\right) \frac{\partial \ln c_{e}}{\partial x}(x, t / 4) \\
& \frac{\partial i_{e}^{ \pm}}{\partial x}(x, t)=a^{ \pm} F j_{n}^{ \pm}(x, t), \\
& j_{n}^{ \pm}(x, t)=\frac{1}{F} i_{0}^{ \pm}(x, t)\left[e^{\frac{\alpha_{a} F}{R T} \eta^{ \pm}(x, t)}-e^{-\frac{\alpha_{c} F}{R T} \eta^{ \pm}(x, t)}\right], \\
& i_{0}^{ \pm}(x, t)=k^{ \pm}\left[c_{s s}^{ \pm}(x, t)\right]^{\alpha_{c}}\left[c_{e}(x, t)\left(c_{s, \max }^{ \pm}-c_{s s}^{ \pm}(x, t)\right)\right]^{\alpha_{a}},
\end{aligned}
$$

$$
\begin{aligned}
\eta^{ \pm}(x, t)= & \phi_{s}^{ \pm}(x, t)-\phi_{e}(x, t) \\
& -U^{ \pm}\left(c_{s s}^{ \pm}(x, t)\right)-F R_{f}^{ \pm} j_{n}^{ \pm}(x, t), \\
c_{s s}^{ \pm}(x, t)= & c_{s}^{ \pm}\left(x, R_{s}^{ \pm}, t\right) .
\end{aligned}
$$

where $D_{e}^{\text {eff }}=D_{e}\left(c_{e}\right) \cdot\left(\varepsilon_{e}^{j}\right)^{\text {brug }}, \sigma^{\text {eff }}=\sigma \cdot\left(\varepsilon_{s}^{j}+\varepsilon_{f}^{j}\right)^{\text {brug }}, \kappa^{\text {eff }}=$ $\kappa\left(c_{e}\right) \cdot\left(\varepsilon_{e}^{j}\right)^{\text {brug }}$ are the effective electrolyte diffusivity, effective solid conductivity, and effective electrolyte conductivity given by the Bruggeman relationship.

The boundary conditions for solid-phase diffusion PDE (1) are

$$
\frac{\partial c_{s}^{ \pm}}{\partial r}(x, 0, t)=0
$$




$$
\frac{\partial c_{s}^{ \pm}}{\partial r}\left(x, R_{s}^{ \pm}, t\right)=-\frac{1}{D_{s}^{ \pm}} j_{n}^{ \pm}(x, t) .
$$

The boundary conditions for the electrolyte-phase diffusion PDE (2) are given by

$$
\begin{aligned}
\frac{\partial c_{e}^{-}}{\partial x}\left(0^{-}, t\right) & =\frac{\partial c_{e}^{+}}{\partial x}\left(0^{+}, t\right)=0 \\
\varepsilon_{e}^{-} D_{e}\left(L^{-}\right) \frac{\partial c_{e}^{-}}{\partial x}\left(L^{-}, t\right) & =\varepsilon_{e}^{\mathrm{sep}} D_{e}\left(0^{\mathrm{sep}}\right) \frac{\partial c_{e}^{\mathrm{sep}}}{\partial x}\left(0^{\mathrm{sep}}, t\right),(1) \\
\varepsilon_{e}^{\mathrm{sep}} D_{e}\left(L^{\mathrm{sep}}\right) \frac{\partial c_{e}^{\mathrm{sep}}}{\partial x}\left(L^{\mathrm{sep}}, t\right) & =\varepsilon_{e}^{+} D_{e}\left(L^{+}\right) \frac{\partial c_{e}^{+}}{\partial x}\left(L^{+}, t\right), \\
c_{e}\left(L^{-}, t\right) & =c_{e}\left(0^{\mathrm{sep}}, t\right) \\
c_{e}\left(L^{\mathrm{sep}}, t\right) & =c_{e}\left(L^{+}, t\right) .
\end{aligned}
$$

The boundary conditions for the electrolyte-phase potential ODE (4) are given by

$$
\begin{aligned}
\phi_{e}\left(0^{-}, t\right) & =0, \\
\phi_{e}\left(L^{-}, t\right) & =\phi_{e}\left(0^{\mathrm{sep}}, t\right), \\
\phi_{e}\left(L^{\mathrm{sep}}, t\right) & =\phi_{e}\left(L^{+}, t\right) .
\end{aligned}
$$

The boundary conditions for the ionic current ODE (5) are given by

$$
i_{e}^{-}\left(0^{-}, t\right)=i_{e}^{+}\left(0^{+}, t\right)=0,
$$

and also note that $i_{e}(x, t)=I(t)$ for $x \in\left[0^{\text {sep }}, L^{\text {sep }}\right]$.

The model input is the applied current density $I(t)\left[\mathrm{A} / \mathrm{m}^{2}\right]$, and the output is the voltage measured across the current collectors,

$$
V(t)=\phi_{s}^{+}\left(0^{+}, t\right)-\phi_{s}^{-}\left(0^{-}, t\right) .
$$

A complete exposition on the model equations and notation can be found in [2], [3]. Symbols are defined in Table III of the Appendix. Note the mathematical structure, which contains linear PDEs (1), quasilinear PDEs (2), ODEs in space (3)-(5), and nonlinear algebraic constraints (6)-(8). This presents a formidable task for model-based state estimation. Consequently, we seek an appropriately reduced model that maintains prediction fidelity - at high C-rates in particular yet enables a provably convergent state observer.

\section{B. SPMe Model Derivation}

The SPMe is derived under the following assumptions:

- [A1]: The solid phase Li concentration in each electrode is constant in spatial coordinate $x$, uniformly in time. Mathematically, $c_{s}^{ \pm}(x, r, t)$ and $j_{n}^{ \pm}(x, t)$ are constant in the $x$ direction.

- [A2]: The exchange current density term $i_{0}^{ \pm}(x, t)$ can be approximated by its averaged value $\bar{i}_{0}^{ \pm}(t)$, which is independent of $x$.

- [A3]: The total moles of lithium in the electrolyte $n_{L i, e}$ and in the solid phase $n_{L i, s}$ are both conserved. This assumption, together with [A1] makes it possible to write the fluxes $j_{n}^{ \pm}(x, t)$ as proportional to current $I(t)$.

- [A4]: The constants $\alpha_{a}=\alpha_{c}$ (hereafter denoted simply by $\alpha$ ). This assumption is almost always true in practice.

These assumptions ultimately render a model consisting of: (i) two linear spherical diffusion PDEs modeling each

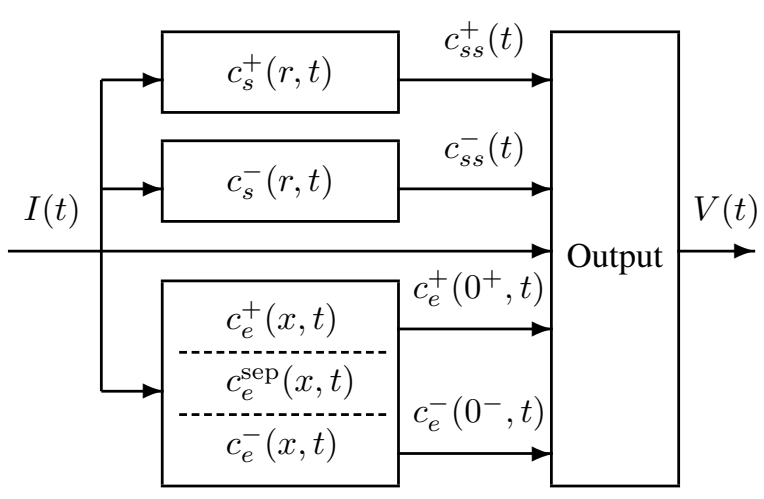

Fig. 2. Block diagram of SPMe. Note that the $c_{s}^{+}, c_{s}^{-}, c_{e}$ subsystems are independent of one another.

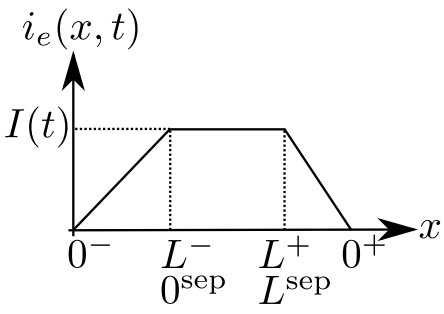

Fig. 3. Simplified form of $i_{e}(x, t)$ in the SPMe model.

electrode's solid concentration dynamics, (ii) a quasilinear diffusion equation (across three domains) modeling the electrolyte concentration dynamics, and (iii) a nonlinear output function mapping boundary values of solid concentration, electrolyte concentration, and current to voltage (see Fig. 2).

We now introduce the resulting SPMe equations. Whenever assumptions [A1]-[A3] remove the spatial dependence of a variable, an overline is added to the variable name to avoid confusion. The first step is to combine [A1], ODE (5) and its boundary conditions (20) to express molar ion flux as proportional to current,

$$
\bar{j}_{n}^{+}(t)=-\frac{I(t)}{F a^{+} L^{+}}, \quad \bar{j}_{n}^{-}(t)=\frac{I(t)}{F a^{-} L^{-}} .
$$

Note the ionic current $i_{e}(x, t)$ has the trapezoidal shape shown in Fig. 3. Apply $\bar{j}_{n}^{ \pm}$in (22) to boundary conditions (10)-(11) and [A1] to derive the solid diffusion equations:

$$
\begin{aligned}
& \frac{\partial \bar{c}_{s}^{ \pm}}{\partial t}(r, t)=\frac{1}{r^{2}} \frac{\partial}{\partial r}\left[D_{s}^{ \pm} r^{2} \frac{\partial \bar{c}_{s}^{ \pm}}{\partial r}(r, t)\right], \\
& \frac{\partial \bar{c}_{s}^{ \pm}}{\partial r}(0, t)=0, \quad \frac{\partial \bar{c}_{s}^{ \pm}}{\partial r}\left(R_{s}^{ \pm}, t\right)= \pm \frac{1}{D_{s}^{ \pm} F a^{ \pm} L^{ \pm}} I(t) .
\end{aligned}
$$

Next, derive the electrolyte diffusion equations by combining PDE (2) with (5), (22), and [A1]:

$$
\begin{aligned}
\frac{\partial c_{e}^{-}}{\partial t}(x, t) & =\frac{\partial}{\partial x}\left[D_{e}\left(c_{e}^{-}\right) \frac{\partial c_{e}^{-}}{\partial x}(x, t)\right]+\frac{\left(1-t_{c}^{0}\right)}{\varepsilon_{e}^{-} F L^{-}} I(t), \\
\frac{\partial c_{e}^{\mathrm{sep}}}{\partial t}(x, t) & =\frac{\partial}{\partial x}\left[D_{e}\left(c_{e}^{\mathrm{sep}}\right) \frac{\partial c_{e}^{\mathrm{sep}}}{\partial x}(x, t)\right]
\end{aligned}
$$




$$
\frac{\partial c_{e}^{+}}{\partial t}(x, t)=\frac{\partial}{\partial x}\left[D_{e}\left(c_{e}^{+}\right) \frac{\partial c_{e}^{+}}{\partial x}(x, t)\right]-\frac{\left(1-t_{c}^{0}\right)}{\varepsilon_{e}^{+} F L^{+}} I(t)
$$

with the same boundary conditions as (12)-(16).

Next, we derive the nonlinear output function for terminal voltage. From (21), we note the voltage $V(t)$ depends on the solid potential at the current collectors $\phi_{s}^{ \pm}(x, t)$. Therefore, we solve (8) in terms of $\phi_{s}$ and spatially averaged quantities,

$$
\phi_{s}^{ \pm}(x, t)=\bar{\eta}^{ \pm}(t)+\bar{\phi}_{e}^{ \pm}(x, t)+U^{ \pm}\left(\bar{c}_{s s}^{ \pm}(t)\right)+F R_{f}^{ \pm} \bar{j}_{n}^{ \pm}(t) .
$$

Next we derive each term on the right hand side of (28). Overpotential $\bar{\eta}^{ \pm}(t)$ is found by solving the Butler-Volmer equation (6) in terms of $\bar{\eta}^{ \pm}(t)$, applying [A1], [A2], [A4], and substituting (22),

$$
\bar{\eta}^{ \pm}(t)=\frac{R T}{\alpha F} \sinh ^{-1}\left(\frac{\mp I(t)}{2 a^{ \pm} L^{ \pm} \bar{i}_{0}^{ \pm}(t)}\right) .
$$

The electrolyte potential $\bar{\phi}_{e}^{ \pm}(x, t)$ is found by integrating ODE (4) w.r.t. $x$ across the entire cell width,

$$
\begin{aligned}
\int_{0^{-}}^{0^{+}} \frac{\partial \phi_{e}}{\partial x}(x, t) d x= & \int_{0^{-}}^{0^{+}} \frac{i_{e}^{ \pm}(x, t)}{\kappa\left(c_{e}\right)} d x+\int_{0^{-}}^{0^{+}} \frac{2 R T}{F}\left(1-t_{c}^{0}\right) \\
& \times\left(1+\frac{d \ln f_{c / a}}{d \ln c_{e}}(x, t)\right) \frac{\partial \ln c_{e}}{\partial x}(x, t) d x .
\end{aligned}
$$

In order to analytically integrate (30), we further assume

- [A5]: The term $k_{f}(x, t) \doteq\left(1+\frac{d \ln f_{c / a}}{d \ln c_{e}}(x, t)\right)$ is approximately constant in $x$, i.e. $\bar{k}_{f}(t) \approx k_{f}(x, t)$.

- [A6]: The term $\kappa\left(c_{e}\right)$ is approximately constant in $c_{e}$, i.e. $\bar{\kappa} \approx \kappa\left(c_{e}\right)$.

This yields the expression

$$
\begin{aligned}
& \bar{\phi}_{e}^{+}\left(0^{+}, t\right)-\bar{\phi}_{e}^{-}\left(0^{-}, t\right)=\frac{L^{+}+2 L^{\mathrm{sep}}+L^{-}}{2 \bar{\kappa}} I(t) \\
& +\frac{2 R T}{F}\left(1-t_{c}^{0}\right) k_{f}(t)\left[\ln c_{e}\left(0^{+}, t\right)-\ln c_{e}\left(0^{-}, t\right)\right] .
\end{aligned}
$$

Now we combine (28)-(31) and (22) to compute $V(t)=$ $\phi_{s}^{+}\left(0^{+}, t\right)-\phi_{s}^{-}\left(0^{-}, t\right)$,

$$
\begin{aligned}
V(t)= & \frac{R T}{\alpha F} \sinh ^{-1}\left(\frac{-I(t)}{2 a^{+} L^{+} \bar{i}_{0}^{+}(t)}\right) \\
- & \frac{R T}{\alpha F} \sinh ^{-1}\left(\frac{I(t)}{2 a^{-} L^{-} \bar{i}_{0}^{-}(t)}\right) \\
+ & U^{+}\left(\bar{c}_{s s}^{+}(t)\right)-U^{-}\left(\bar{c}_{s s}^{-}(t)\right)-\left(\frac{R_{f}^{+}}{a^{+} L^{+}}+\frac{R_{f}^{-}}{a^{-} L^{-}}\right) I(t) \\
+ & \frac{L^{+}+2 L^{\mathrm{sep}}+L^{-}}{2 \bar{\kappa}} I(t) \\
+ & k_{\mathrm{conc}}(t)\left[\ln c_{e}\left(0^{+}, t\right)-\ln c_{e}\left(0^{-}, t\right)\right] \\
& V(t)=h\left(\bar{c}_{s s}^{+}, \bar{c}_{s s}^{-}, c_{e}^{+}\left(0^{+}, t\right), c_{e}^{-}\left(0^{-}, t\right), I(t)\right),
\end{aligned}
$$

where $k_{\text {conc }}=\frac{2 R T}{F}\left(1-t_{c}^{0}\right) k_{f}(t)$.

This summarizes the SPMe. Note the significantly simplified structure. The dynamical equations (23), (25)-(27) are linear and quasilinear PDEs, respectively. The boundary conditions are all linear. Finally, the output function (32) is nonlinear w.r.t. states and inputs. As shown in Section III, the SPMe is amenable to state observer design with provable convergence. Moreover, it maintains accuracy at high C-rates, as discussed in Section II-C.

Remark 1 (SPMe vs. SPM Comparison). Note the voltage expression (32) is identical to the SPM voltage expression [15], but the SPMe adds the last two terms. These respectively represent ohmic potential drop due electrolyte conductivity and the electrolyte concentration overpotential.

Remark 2 (Comparison to existing SPMe Models). The SPMe model development was motivated by the difficulty of extending the results in [15] using the models available in the literature. For instance, the model in [19] does not allow for the output-inversion step we require to design a provably convergent observer, due to the spatial distribution of the surface concentration in the solid given by the interaction of the approximate diffusion representation and the polynomial (in space) approximation of the electrolyte concentration profiles.

The model in [21] linearizes the transportation equations first. Then it applies a Laplace transformation and performs a polynomial approximation across space. This results in a linear output function, but produces a non-physical state-space representation. The model we propose, in contrast, maintains the physical interpretation of the state-space - a useful property for state estimation. Furthermore, our time-domain model can accommodate some time-varying coefficients in a much simpler way than an approximate transfer function model.

The models in [18], [20], [22] are the most similar to the SPMe derived here, as they apply [A1] in addition to various numerical approximations. In [18], bulk solid concentration is used in the voltage output function instead of the surface concentration we use here (see (26) in [18]). In the case of [20], volume averaging is performed in the electrolyte phase which partially obscures electrolyte polarization. In [22], the authors use an approximation of the solid state diffusion equation instead of retaining the PDE version we use in (23)(24) (see Section 2 of [22]). Since our main objective is provably convergent state observers, we additionally analyze the SPMe's dynamical properties in Section II-D. Furthermore, the structure of our proposed SPMe would allow for relatively simple extensions to nonhomogeneous (in space) transport and conductivity terms, albeit rendering the integration required to obtain the output equation in (32) harder. Namely, it would require numerically integrating the electrolyte potential gradient instead of obtaining an explicit form for the ohmic potential drop.

TABLE I

RMS VOLTAGE ERROR WITH RESPECT TO DFN MODEL

\begin{tabular}{c|ccccc|c}
\hline \hline & $0.1 \mathrm{C}$ & $0.5 \mathrm{C}$ & $1 \mathrm{C}$ & $2 \mathrm{C}$ & $5 \mathrm{C}$ & UDDSx2 \\
\hline SPM & $2 \mathrm{mV}$ & $8 \mathrm{mV}$ & $17 \mathrm{mV}$ & $31 \mathrm{mV}$ & $72 \mathrm{mV}$ & $14 \mathrm{mV}$ \\
SPMe & $2 \mathrm{mV}$ & $5 \mathrm{mV}$ & $9 \mathrm{mV}$ & $13 \mathrm{mV}$ & $19 \mathrm{mV}$ & $7 \mathrm{mV}$ \\
\hline \hline
\end{tabular}




\section{Model Comparison}

In this subsection, we compare voltage predictions between the SPMe, SPM, and DFN models. Note the SPM output voltage equation is equivalent to (32), but without the last two terms. The model parameters used in this study originate from the publicly available DUALFOIL simulation package [30] and correspond to a lithium cobalt oxide cathode / graphite anode chemistry. Discharge curves at various $\mathrm{C}$-rates are provided in Fig. 4(a). At low C-rates (e.g. 0.1C or 0.5C) the electrolyte concentration gradient is negligible and both the SPM and SPMe accurately predict voltage. As C-rate increases beyond $0.5 \mathrm{C}$ the electrolyte gradient becomes significant (c.f. Fig. 4(b)), thereby violating the SPM model reduction assumption. Consequently, the SPMe predicts voltage with greater accuracy than the SPM (see Table I). Figure 5 provides a visual comparison of the SPM, SPMe, and DFN models on a transient electric vehicle-like charge/discharge cycle, generated from two concatenated Urban Dynamometer Driving Schedules (UDDSx2) (see [31] for details). The mean C-rate is about $0.5 \mathrm{C}$. The SPM predicts voltage with $14 \mathrm{mV}$ RMS error, whereas the SPMe achieves $7 \mathrm{mV}$ RMS error. Clearly, battery management systems for applications with large sustained Crates greatly benefit from models with electrolyte dynamics. This, however, complicates observer design - a challenge we address in this manuscript.

\section{Mathematical Model Properties}

The causal structure of the SPMe is elucidated in Fig. 2. Namely, the $c_{s}^{+}, c_{s}^{-}, c_{e}$ subsystems are all mutually independent from one another. Moreover, they are governed by linear $\left(c_{s}^{ \pm}\right.$subsystem) or quasilinear ( $c_{e}$ subsystems) PDEs. Note the bar notation used in the previous section is herein after dropped to reduce clutter. Note also that the PDE subsystems produce boundary values $c_{s s}^{+}(t), c_{s s}^{-}(t), c_{e}^{+}\left(0^{+}, t\right), c_{e}^{-}\left(0^{-}, t\right)$ that serve as inputs to the nonlinear output function. We pursue the following observer structure, which exploits the linear PDE dynamical subsystems and nonlinear output function. (i) Design linear observers for each dynamical subsystem, assuming a "virtual" measurement of $c_{s s}^{+}(t)$. (ii) Design a recursive nonlinear output function inversion scheme to "process" $c_{s s}^{+}(t)$ from measurements $I(t), V(t)$.

The model is also characterized by the following dynamical properties, which are the critical insights underpinning the observer design in Section III. We present the following propositions, whose proofs are straight-forward, non-insightful within themselves, and omitted for brevity.

Proposition 1 (Marginal stability of $c_{s}^{+}, c_{s}^{-}, c_{e}$ subsystems). Each individual subsystem in (23)-(27) governing states $c_{s}^{+}(r, t), c_{s}^{-}(r, t)$, and $c_{e}(x, t)$ is marginally stable. Specifically, each subsystem contains one eigenvalue at the origin, and the remaining eigenvalues lie on the negative real axis of the complex plane.

Proposition 2 (Conservation of solid-phase lithium). The moles of lithium in the solid phase is conserved [25]. Mathe-
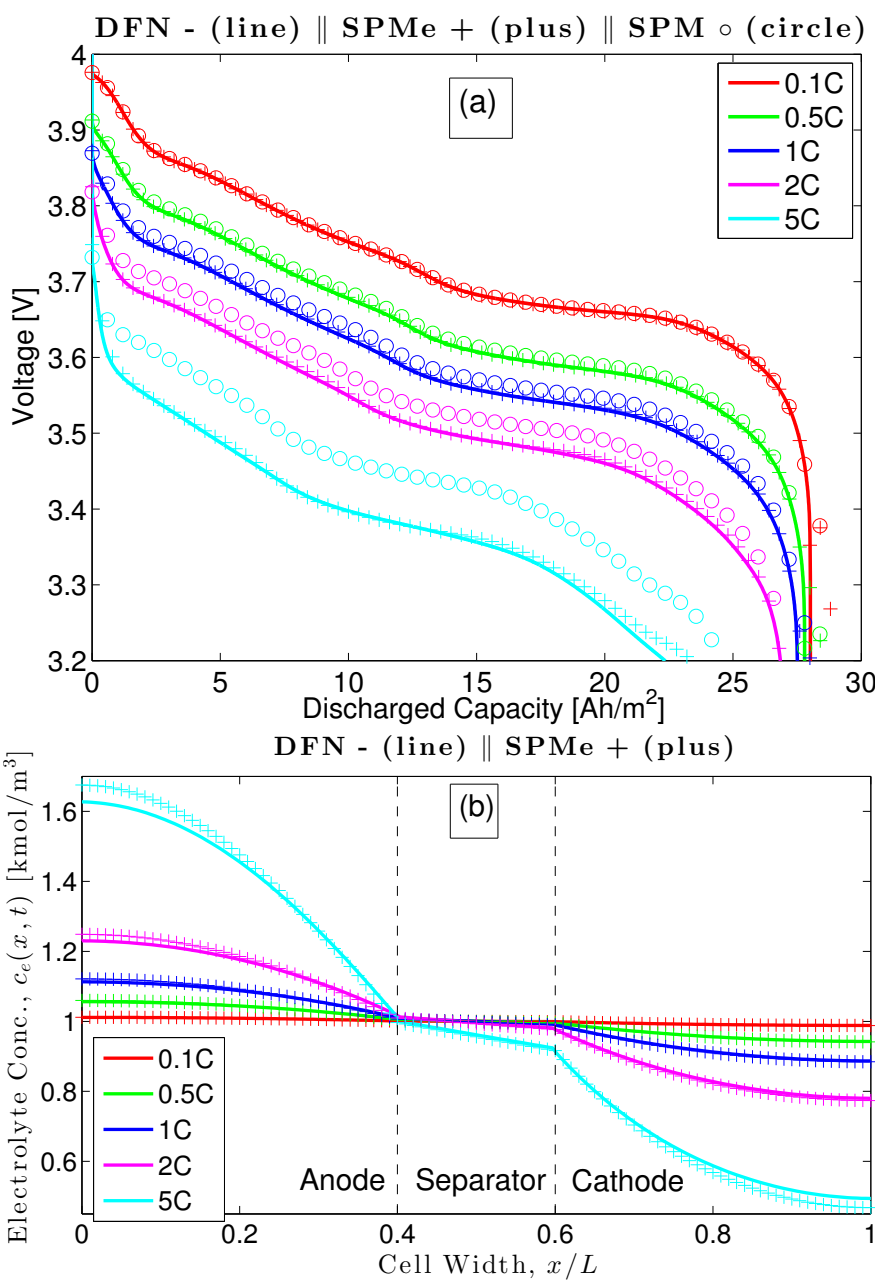

Fig. 4. [a] Voltage discharge curves across various C-rates, for the single particle model (SPM) [15], single particle model with electrolyte (SPMe), and Doyle-Fuller-Newman (DFN) model [30]. The SPMe maintains smaller errors as C-rate increases relative to the SPM. [b] Electrolyte concentration $c_{e}(x, t)$ predicted by DFN and SPMe for various C-rates after $5 \mathrm{Ah} / \mathrm{m}^{2}$ of discharge. The concentration gradient increases as $\mathrm{C}$-rate increases.

matically, $\frac{d}{d t}\left(n_{L i, s}(t)\right)=0$ where

$$
n_{L i, s}(t)=\sum_{j \in\{+,-\}} \frac{\varepsilon_{s}^{j} L^{j}}{\frac{4}{3} \pi\left(R_{s}^{j}\right)^{3}} \int_{0}^{R_{s}^{j}} 4 \pi r^{2} c_{s}^{j}(r, t) d r .
$$

In the following observer design, we select the estimation gains to conserve moles of lithium.

Proposition 3 (Conservation of electrolyte-phase lithium). The moles of lithium in the electrolyte phase is conserved. Mathematically, $\frac{d}{d t}\left(n_{L i, e}(t)\right)=0$ where

$$
n_{L i, e}(t)=\sum_{j \in\{-, s e p,+\}} \varepsilon_{e}^{j} \int_{0^{j}}^{L^{j}} c_{e}^{j}(x, t) d x .
$$

In particular, this property implies that the equilibrium solution of the $c_{e}$ subsystem (25)-(27) with zero current density, i.e. $I(t)=0$, is given by

$$
c_{e, e q}(x)=\frac{n_{L i, e}}{\varepsilon_{e}^{-} L^{-}+\varepsilon_{e}^{\mathrm{sep}} L^{\mathrm{sep}}+\varepsilon_{e}^{+} L^{+}}, \forall x \in\left[0^{-}, 0^{+}\right] .
$$




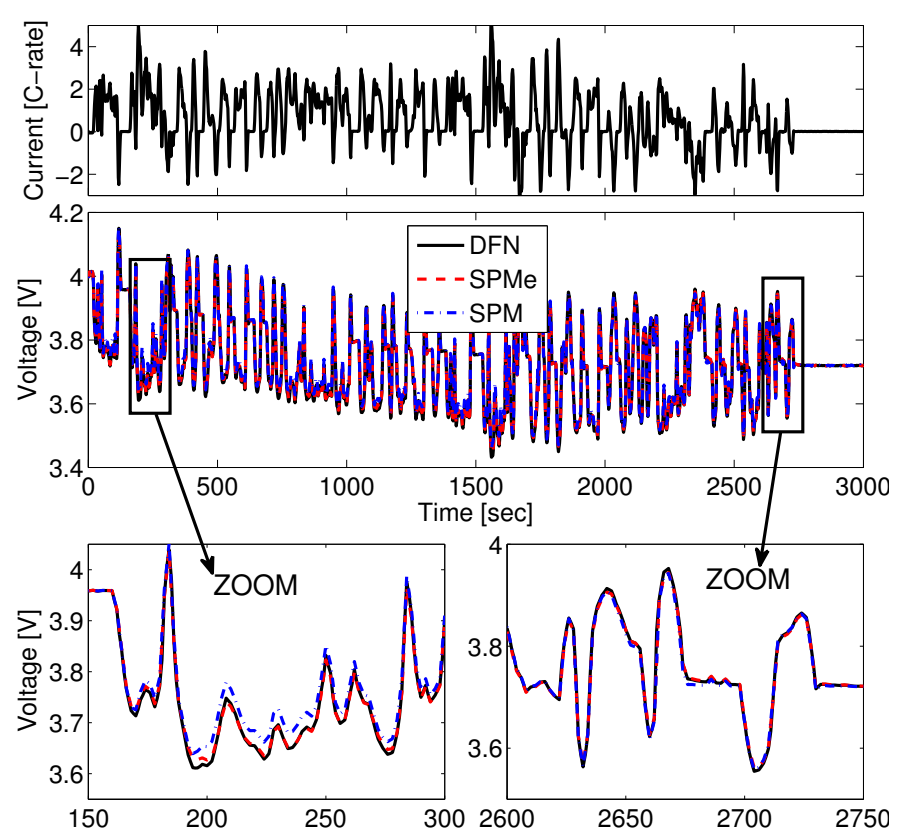

Fig. 5. Comparison of SPM, SPMe, and DFN model predictions on two concatenated UDDS charge/discharge cycles.

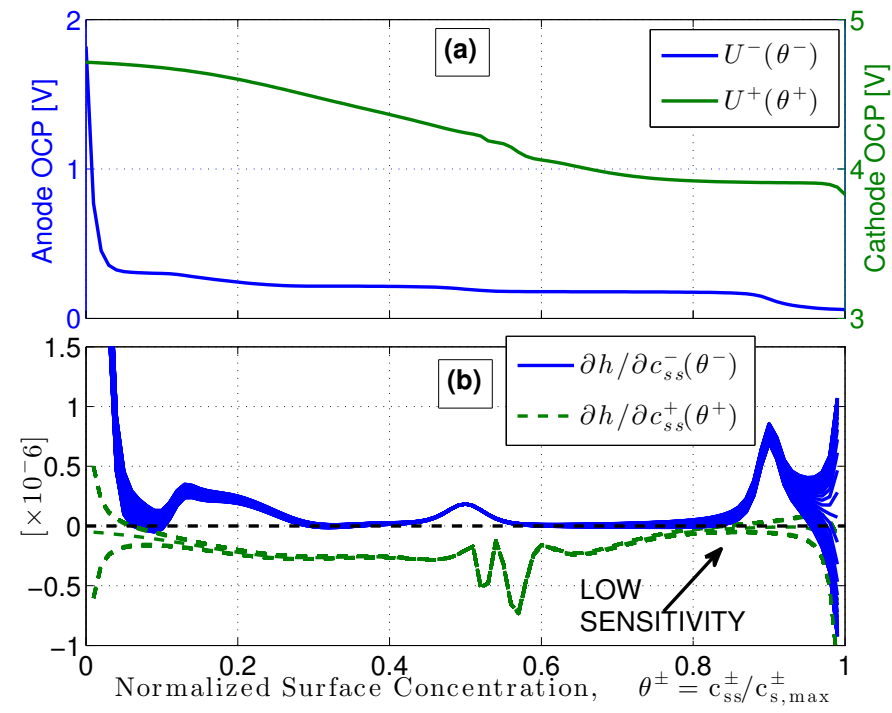

Fig. 6. (a) Open circuit potential functions $U^{-}\left(\theta^{-}\right), U^{+}\left(\theta^{+}\right)$; and (b) Gradients of $h\left(c_{s s}^{+}, c_{s s}^{-}, I\right)$ in (33), as functions of normalized surface concentration $\theta^{ \pm}=c_{s s}^{ \pm} / c_{s, \text { max }}^{ \pm}$, for currents ranging from $-5 \mathrm{C}$ to $+5 \mathrm{C}$.

\section{E. Invertibility Analysis}

Next, we study invertibility of the output function (32) w.r.t. boundary state variables $c_{s s}^{ \pm}(t)$. Invertibility enables one to "process" surface concentration from measurements $I(t), V(t)$ and design linear observers. Let output function $h: \mathbb{R} \times \mathbb{R} \times \mathbb{R} \rightarrow \mathbb{R}$ be defined such that $V(t)=h\left(c_{s s}^{+}, c_{s s}^{-}, I\right)$, where we assume the electrolyte concentrations $c_{e}^{ \pm}\left(0^{ \pm}, t\right)$ in (33) are known and therefore suppress their dependence. This assumption is valid, because we later substitute exponentially convergent estimates of $c_{e}^{ \pm}\left(0^{ \pm}, t\right)$ into output function $h(\cdot)$ -

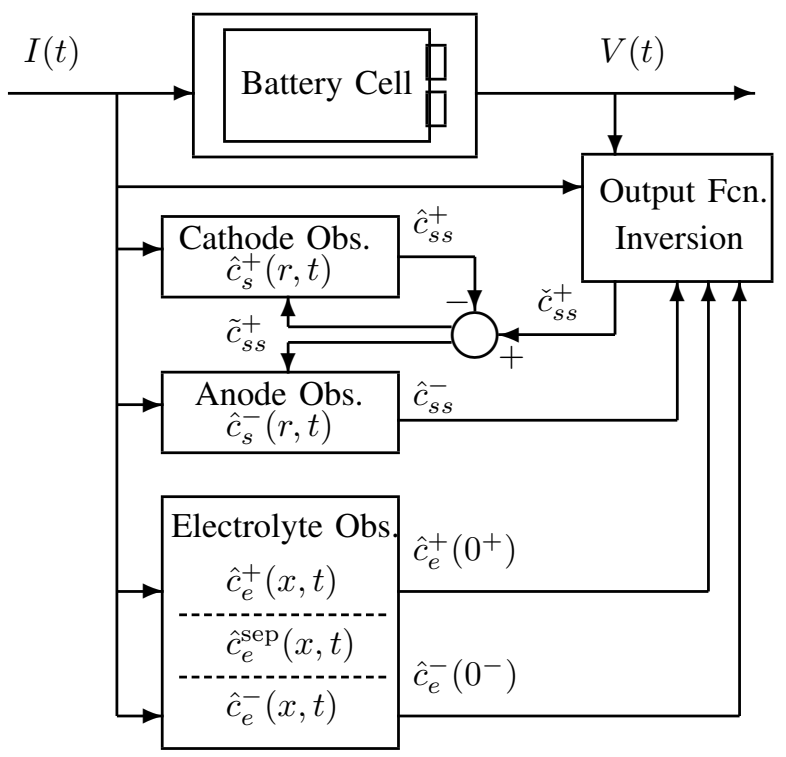

Fig. 7. Block diagram of SPMe Observer.

as shown in Section IV. The output function $h\left(c_{s s}^{+}, c_{s s}^{-}, \cdot\right)$ is locally invertible around $c_{s}^{+}=a$ or $c_{s}^{-}=b$, if $h\left(c_{s s}^{+}, c_{s s}^{-}, \cdot\right)$ is one-to-one in the respective local domains $\left\{c_{s s}^{+}:\left|c_{s s}^{+}-a\right|<\epsilon\right\}$ or $\left\{c_{s s}^{-}:\left|c_{s s}^{-}-b\right|<\epsilon\right\}$, for small $\epsilon>0$. If the aforementioned condition is true for all $I$, then $h(a, b, \cdot)$ is locally invertible uniformly in $I$. Consequently, invertibility can be tested by examining the gradient of $h\left(c_{s s}^{+}, c_{s s}^{-}, \cdot\right)$ w.r.t. $c_{s s}^{+}$or $c_{s s}^{-}$.

Under equilibrium conditions and zero current, the output function simplifies to

$$
V(t)=U^{+}\left(c_{s s}^{+}(t)\right)-U^{-}\left(c_{s s}^{-}(t)\right) .
$$

Figure 6(a) provides the open circuit potential (OCP) functions $U^{-}\left(\theta^{-}\right), U^{+}\left(\theta^{+}\right)$where $\theta^{ \pm}=c_{s s}^{ \pm} / c_{s, \text { max }}^{ \pm}$. In general, $U^{+}\left(\theta^{+}\right)$is more sensitive to perturbations in its argument than $U^{-}\left(\theta^{-}\right)$. Figure $6(\mathrm{~b})$ depicts the partial derivatives of $h\left(c_{s s}^{+}, c_{s s}^{-}, I\right)$ in (33) w.r.t. $c_{s s}^{-}$and $c_{s s}^{+}$at equilibrium conditions, for currents ranging from $-5 \mathrm{C}$ to $+5 \mathrm{C}$. In general, $h$ is non-decreasing w.r.t. $c_{s s}^{-}$and is non-increasing w.r.t. $c_{s s}^{+}$. It is important to note that $h$ is strictly monotonically decreasing w.r.t. $c_{s s}^{+}$over a larger range than $h$ is strictly monotonically increasing w.r.t. $c_{s s}^{-}$. This property is critical, since it demonstrates that voltage is generally more sensitive to perturbations in cathode surface concentration than anode surface concentration. Consequently, we opt to pursue output function inversion w.r.t. $c_{s s}^{+}$instead of $c_{s s}^{-}$. Note $\partial h / \partial c_{s s}^{+} \approx 0$ for $0.8 \leq \theta^{+} \leq 0.9$. This region is a "blind spot" with respect to output inversion, a limitation we explore by analysis in Section IV-B and by simulation in Section V.

\section{State Observer Design}

The observer design process is summarized as follows:

1) Perform dimension normalization and state transformation on cathode dynamics.

2) Design a backstepping PDE observer for the transformed cathode dynamics. 
3) Perform inverse state transformation and unnormalization.

4) Design anode observer to conserve lithium in solid.

5) Design an open-loop electrolyte observer.

6) Design an output function inversion scheme.

\section{A. Normalization and State Transformation}

First we perform normalization and state transformation to simplify the mathematical structure of the cathode observer. The radial $r$ and time $t$ coordinates are scaled as follows

$$
\bar{r}=\frac{r}{R_{s}^{+}}, \quad \bar{t}=\frac{D_{s}^{+}}{\left(R_{s}^{+}\right)^{2}} t .
$$

Henceforth we will drop the bars over the space and time coordinates to simplify notation. Next we perform a state transformation to eliminate the first spatial derivative in the spherical diffusion equation (23). Namely, let

$$
c(r, t)=r c_{s}^{+}(r, t) .
$$

This normalization and state transformation produces the following PDE with Dirichlet and Robin boundary conditions

$$
\begin{aligned}
\frac{\partial c}{\partial t}(r, t) & =\frac{\partial^{2} c}{\partial r^{2}}(r, t), \\
c(0, t) & =0, \\
\frac{\partial c}{\partial r}(1, t)-c(1, t) & =\rho I(t) .
\end{aligned}
$$

The parameter $\rho=R_{s}^{+} /\left(D_{s}^{+} F a^{+} L^{+}\right)$groups together known parameters.

\section{B. Cathode Backstepping Observer}

The cathode state estimator subsystem structure consists of a copy of the plant (40)-(42) plus boundary state error injection, as follows

$$
\begin{aligned}
\frac{\partial \hat{c}}{\partial t}(r, t) & =\frac{\partial^{2} \hat{c}}{\partial r^{2}}(r, t)+p^{+}(r)[c(1, t)-\hat{c}(1, t)] \\
\hat{c}(0, t) & =0 \\
\frac{\partial \hat{c}}{\partial r}(1, t)-\hat{c}(1, t) & =\rho I(t)+p_{0}^{+}[c(1, t)-\hat{c}(1, t)]
\end{aligned}
$$

Following the procedure in [32], [15], the observer gains are

$$
\begin{aligned}
p^{+}(r) & =\frac{-\lambda r}{2 z}\left[I_{1}(z)-\frac{2 \lambda}{z} I_{2}(z)\right], \\
\text { where } \quad z & =\sqrt{\lambda\left(r^{2}-1\right)}, \\
p_{0}^{+} & =\frac{1}{2}(3-\lambda), \quad \text { for } \quad \lambda<\frac{1}{4},
\end{aligned}
$$

and $I_{1}(z)$ and $I_{2}(z)$ are, respectively, the first and second order modified Bessel functions of the first kind. Parameter $\lambda$ governs the error system eigenvalue locations.

\section{Inverse Transformation and Un-normalization}

Next we re-write the cathode observer (43)-(45) into the original coordinates $\hat{c}_{s}^{+}$by inverting transformation (39) and un-normalizing the dimensions (38). The final result is

$$
\begin{aligned}
\frac{\partial \hat{c}_{s}^{+}}{\partial t}(r, t)=D_{s}^{+} & {\left[\frac{2}{r} \frac{\partial \hat{c}_{s}^{+}}{\partial r}(r, t)+\frac{\partial^{2} \hat{c}_{s}^{+}}{\partial r^{2}}(r, t)\right] } \\
& +\bar{p}^{+}(r)\left[c_{s s}^{+}(t)-\hat{c}_{s s}^{+}(t)\right], \\
\frac{\partial \hat{c}_{s}^{+}}{\partial r}(0, t)= & 0, \\
\frac{\partial \hat{c}_{s}^{+}}{\partial r}\left(R_{s}^{+}, t\right)= & \frac{I(t)}{D_{s}^{+} F a^{+} L^{+}}+\bar{p}_{0}^{+}\left[c_{s s}^{+}(t)-\hat{c}_{s s}^{+}(t)\right] .
\end{aligned}
$$

where the observer gains are

$$
\begin{aligned}
\bar{p}^{+}(r) & =\frac{-\lambda D_{s}^{+}}{2 R_{s}^{+} \bar{z}}\left[I_{1}(\bar{z})-\frac{2 \lambda}{\bar{z}} I_{2}(\bar{z})\right], \\
\text { where } \bar{z} & =\sqrt{\lambda\left(\frac{r^{2}}{\left(R_{s}^{+}\right)^{2}}-1\right)}, \\
\bar{p}_{0}^{+} & =\frac{1}{2 R_{s}^{+}}(3-\lambda), \quad \text { for } \quad \lambda<\frac{1}{4} .
\end{aligned}
$$

\section{Anode Observer}

Now we focus on an observer for the anode subsystem. Our objective is to design the observer gains such that the total moles of solid-phase lithium are conserved. The anode state estimator subsystem structure consists of a copy of the plant (23) plus cathode surface concentration error injection, as follows

$$
\begin{aligned}
\frac{\partial \hat{c}_{s}^{-}}{\partial t}(r, t)=D_{s}^{-} & {\left[\frac{2}{r} \frac{\partial \hat{c}_{s}^{-}}{\partial r}(r, t)+\frac{\partial^{2} \hat{c}_{s}^{-}}{\partial r^{2}}(r, t)\right] } \\
& +\bar{p}^{-}(r)\left[c_{s s}^{+}(t)-\hat{c}_{s s}^{+}(t)\right], \\
\frac{\partial \hat{c}_{s}^{-}}{\partial r}(0, t)= & 0, \\
\frac{\partial \hat{c}_{s}^{-}}{\partial r}\left(R_{s}^{-}, t\right)= & \frac{I(t)}{D_{s}^{-} F a^{-} L^{-}}+\bar{p}_{0}^{-}\left[c_{s s}^{+}(t)-\hat{c}_{s s}^{+}(t)\right] .
\end{aligned}
$$

We seek to design $\bar{p}^{-}(r), \bar{p}_{0}^{-}$such that $\frac{d}{d t}\left(\hat{n}_{L i, s}(t)\right)=0$. This property holds true under the following relations between the estimation gains

$$
\begin{array}{r}
a^{+} L^{+} D_{s}^{+} \bar{p}_{0}^{+}+a^{-} L^{-} D_{s}^{-} \bar{p}_{0}^{-}=0, \\
\frac{a^{+} L^{+}}{\left(R_{s}^{+}\right)^{2}} \int_{0}^{R_{s}^{+}} r^{2} \bar{p}^{+}(r) d r+\frac{a^{-} L^{-}}{\left(R_{s}^{-}\right)^{2}} \int_{0}^{R_{s}^{-}} r^{2} \bar{p}^{-}(r) d r=0 .
\end{array}
$$

Imposing the structure $\bar{p}^{-}(r)=\bar{p}^{-}$for $r \in\left[0, R_{s}^{+}\right]$, we obtain the anode gains in terms of the cathode gains,

$$
\begin{aligned}
& \bar{p}_{0}^{-}=-\frac{a^{+} L^{+} D_{s}^{+}}{a^{-} L^{-} D_{s}^{-}} \bar{p}_{0}^{+}, \\
& \bar{p}^{-}=-\frac{a^{+} L^{+}}{\left(R_{s}^{+}\right)^{2} \varepsilon_{s}^{-} L^{-}} \int_{0}^{R_{s}^{+}} r^{2} \bar{p}^{+}(r) d r,
\end{aligned}
$$

where $\varepsilon_{s}^{-}=3 /\left(a^{-} R_{s}^{-}\right)$. 


\section{E. Solid Phase Initial Estimate}

The observer is initialized according to the assumption:

- [A7]: The total moles of lithium in the solid phase, $n_{L i, s}$ in (34), is known beforehand.

Therefore, the initial conditions for the composite observer (55)-(57), (49)-(51) must verify

$$
n_{L i, s}=\sum_{j \in\{+,-\}} \frac{\varepsilon_{s}^{j} L^{j}}{\frac{4}{3} \pi\left(R_{s}^{j}\right)^{3}} \int_{0}^{R_{s}^{j}} 4 \pi r^{2} \hat{c}_{s}^{j}(r, 0) d r,
$$

which has the physical interpretation that the observer and plant have identical total moles of lithium in the solid, uniformly in time. If we further consider initial estimates corresponding to a steady-state condition, i.e. the concentrations are uniform in $r$, then (62) simplifies to

$$
n_{L i, s}=\varepsilon_{s}^{+} L^{+} \hat{c}_{s, 0}^{+}+\varepsilon_{s}^{-} L^{-} \hat{c}_{s, 0}^{-} .
$$

where $\hat{c}_{s, 0}^{+}, \hat{c}_{s, 0}^{-}$are the initial estimates corresponding to steady-state. In practice, the value of $n_{L i, s}$ might be provided by the cell maker, or identified via offline model parameterization schemes (e.g. [33], [34]), or online capacity estimators (e.g. [15], [35]). We examine relaxing [A7] in Section V-C.

\section{F. Electrolyte Observer}

For the electrolyte subsystems, we apply an open-loop observer of the form,

$$
\begin{aligned}
\frac{\partial \hat{c}_{e}^{-}}{\partial t}(x, t) & =\frac{\partial}{\partial x}\left[D_{e} \frac{\partial \hat{c}_{e}^{-}}{\partial x}(x, t)\right]+\frac{1-t_{c}^{0}}{\varepsilon_{e}^{-} F L^{-}} I(t), \\
\frac{\partial \hat{c}_{e}^{\mathrm{sep}}}{\partial t}(x, t) & =\frac{\partial}{\partial x}\left[D_{e} \frac{\partial \hat{c}_{e}^{\mathrm{sep}}}{\partial x}(x, t)\right], \\
\frac{\partial \hat{c}_{e}^{+}}{\partial t}(x, t) & =\frac{\partial}{\partial x}\left[D_{e} \frac{\partial \hat{c}_{e}^{+}}{\partial x}(x, t)\right]-\frac{1-t_{c}^{0}}{\varepsilon_{e}^{+} F L^{+}} I(t),
\end{aligned}
$$

with boundary conditions

$$
\begin{aligned}
& \frac{\partial \hat{c}_{e}^{-}}{\partial x}\left(0^{-}, t\right)=\frac{\partial \hat{c}_{e}^{+}}{\partial x}\left(0^{+}, t\right)=0, \\
& \varepsilon_{e}^{-} D_{e}\left(L^{-}\right) \frac{\partial \hat{c}_{e}^{-}}{\partial x}\left(L^{-}, t\right)=\varepsilon_{e}^{\mathrm{sep}} D_{e}\left(0^{\mathrm{sep}}\right) \frac{\partial \hat{c}_{e}^{\mathrm{sep}}}{\partial x}\left(0^{\mathrm{sep}}, t\right), \\
& \varepsilon_{e}^{\mathrm{sep}} D_{e}\left(L^{\mathrm{sep}}\right) \frac{\partial \hat{c}_{e}^{\mathrm{sep}}}{\partial x}\left(L^{\mathrm{sep}}, t\right)=\varepsilon_{e}^{+} D_{e}\left(L^{+}\right) \frac{\partial \hat{c}_{e}^{+}}{\partial x}\left(L^{+}, t\right), \\
& \hat{c}_{e}^{-}\left(L^{-}, t\right)=\hat{c}_{e}^{\mathrm{sep}}\left(0^{\mathrm{sep}}, t\right), \\
& \hat{c}_{e}^{\mathrm{sep}}\left(L^{\mathrm{sep}}, t\right)=\hat{c}_{e}^{+}\left(L^{+}, t\right) .
\end{aligned}
$$

The observer is initialized according to the following assumption.

- [A8]: The total moles of lithium in the electrolyte, $n_{L i, e}$ in (35), is known beforehand.

Therefore, the initial conditions for the electrolyte observer (64)-(71) must verify

$$
n_{L i, e}=\sum_{j \in\{-, \mathrm{sep},+\}} \varepsilon_{e}^{j} \int_{0^{j}}^{L^{j}} \hat{c}_{e}^{j}(x, 0) d x,
$$

which has the physical interpretation that the observer and plant have identical total moles of lithium in the electrolyte, uniformly in time. If we further consider initial estimates corresponding to a steady-state condition, i.e. the concentrations are uniform in $x$, then (72) simplifies to

$$
\hat{c}_{e}^{j}(x, 0)=\frac{n_{L i, e}}{\varepsilon_{e}^{-} L^{-}+\varepsilon_{e}^{\mathrm{sep}} L^{\mathrm{sep}}+\varepsilon_{e}^{+} L^{+}}, j \in\{-, \operatorname{sep},+\} .
$$

In practice, the value of $n_{L i, e}$ might be provided by the cell maker, or identified via offline model parameterization schemes, e.g. [33], [34]. We examine relaxing [A8] in Section V-D.

\section{G. Output Function Inversion}

In Sections III-A to III-D, we designed linear state observers assuming access to surface concentration (i.e. boundary value) $c_{s s}^{+}(t)$. In this subsection we develop a nonlinear gradient algorithm to compute $c_{s s}^{+}(t)$ from measurements $I(t), V(t)$ by inverting the nonlinear output function (32).

To focus on the task at hand, we re-write the output function as

$$
V(t)=h\left(c_{s s}^{+}, t\right)
$$

where the dependence on $c_{s s}^{-}(t), c_{e}^{-}\left(0^{-}, t\right), c_{e}^{+}\left(0^{+}, t\right)$ has been suppressed into a singular dependence on $t$. Denote $\check{c}_{s s}^{+}(t)$ as the "processed" cathode surface concentration resulting from this output function inversion procedure. Then the error between the true and processed values is $\tilde{\theta}=c_{s s}^{+}-\check{c}_{s s}^{+}$. Now re-write (74) as

$$
V(t)=h\left(\tilde{\theta}+\check{c}_{s s}^{+}, t\right)
$$

Next, we approximate the right-hand side of (75) using a firstorder Taylor series with respect to $\tilde{\theta}$ about $\tilde{\theta}=0$,

$$
V(t) \approx h\left(\check{c}_{s s}^{+}, t\right)+\frac{\partial h}{\partial c_{s s}^{+}}\left(\check{c}_{s s}^{+}, t\right) \cdot \tilde{\theta} .
$$

Define inversion error and regressor signals $e_{n l}(t)$ and $\phi(t)$, respectively, as

$$
e_{n l}(t)=V(t)-h\left(\check{c}_{s s}^{+}, t\right), \quad \phi(t)=\frac{\partial h}{\partial c_{s s}^{+}}\left(\check{c}_{s s}^{+}, t\right) .
$$

The approximated output function can now be written in regressor form as $e_{n l}(t)=\phi(t) \tilde{\theta}$. A gradient update law for $\check{c}_{s s}^{+}(t)$ that minimizes $\frac{1}{2} \gamma e_{n l}^{2}(t)$ is given by [36]:

$$
\frac{d}{d t} \check{c}_{s s}^{+}(t)=\gamma \phi e_{n l} .
$$

Note that gain $\gamma$ is a user-selected parameter to trade-off convergence speed and sensitivity to noise.

Remark 3. The update law (78) can be embellished in two practically useful ways. First, a nonlinear least squares update can be applied to dynamically determine the gain parameter (see $\S 4.3$ of [36]). Second, the projection operator can be applied to constrain $\check{c}_{s s}^{+}(t)$ within the set $\left[c_{s, \min }^{+}, c_{s, \max }^{+}\right]$(see $\S 4.4$ of [36]). 


\section{H. Summary of Observer Design}

The complete SPMe observer design is summarized in Table II. It summarizes each subsystem with a reference to the appropriate differential equations. Note the observer has only two scalar design parameters, $\lambda$ and $\gamma$. Parameter $\lambda$ governs the eigenvalues of the cathode observer subsystem, and $\gamma$ governs the output inversion convergence speed. Kalman filter (KF) based observers, in contrast, typically have much more than 2 parameters, depending on the discretization method. Specifically, KFs have up to $\left(n_{x}^{2}+n_{x}\right) / 2+\left(n_{y}^{2}+n_{y}\right) / 2$ tuning parameters, where $n_{x}$ and $n_{y}$ are the number of states and measurements, respectively. The small number of tuning parameters is an additional benefit of the proposed observer design, in addition to provable stability properties.

TABLE II

SUMMARY OF SPME OBSERVER EQUATIONS

\begin{tabular}{l|c|c|c}
\hline \hline Subsystem & State Vars. & Diff. Eqns. & Design Params. \\
\hline $\begin{array}{l}\text { Cathode, } \\
\text { solid phase }\end{array}$ & $\hat{c}_{s s}^{+}(r, t)$ & $(49)-(54)$ & \\
$\begin{array}{l}\text { Anode, } \\
\text { solid phase }\end{array}$ & $\hat{c}_{s s}^{-}(r, t)$ & $(55)-(57),(60)-(61)$ & $\lambda$ in (52)-(54) \\
\hline Electrolyte & $\begin{array}{c}\hat{c}_{e}^{-}(x, t) \\
\hat{c}_{e}^{\text {sep }}(x, t)\end{array}$ & $(64)-(71)$ & none \\
& $\hat{c}_{e}^{+}(x, t)$ & $(77)-(78)$ & $\gamma$ in (78) \\
\hline $\begin{array}{l}\text { Output Fcn. } \\
\text { Inversion }\end{array}$ & $\check{c}_{s s}^{+}(t)$ & & \\
\hline \hline
\end{tabular}

\section{Stability Analysis}

In this section, we prove stability of the composite observer, comprised of the solid phase and electrolyte phase subsystems, assuming measurements of cathode surface concentration $c_{s s}^{+}(t)$. We also prove convergence of the output inversion scheme under an appropriate monotonicity assumption.

\section{A. Solid + Electrolyte Phase Estimation Error Stability}

Consider the cathode $\left(\tilde{c}_{s}^{+}\right)$and anode $\left(\tilde{c}_{s}^{-}\right)$estimation error subsystems, where $\tilde{c}_{s}^{+}=c_{s}^{+}-\hat{c}_{s}^{+}$and $\tilde{c}_{s}^{-}=c_{s}^{-}-\hat{c}_{s}^{-}$,

$$
\frac{\partial \tilde{c}_{s}^{+}}{\partial t}(r, t)=D_{s}^{+}\left[\frac{2}{r} \frac{\partial \tilde{c}_{s}^{+}}{\partial r}(r, t)+\frac{\partial^{2} \tilde{c}_{s}^{+}}{\partial r^{2}}(r, t)\right]-\bar{p}^{+}(r) \tilde{c}_{s s}^{+}(t),
$$$$
\frac{\partial \tilde{c}_{s}^{+}}{\partial r}(0, t)=0, \quad \frac{\partial \tilde{c}_{s}^{+}}{\partial r}\left(R_{s}^{+}, t\right)=-\bar{p}_{0}^{+} \tilde{c}_{s s}^{+}(t),
$$$$
\tilde{c}_{s s}^{+}(r, 0)=c_{s s}^{+}(r, 0)-\hat{c}_{s s}^{+}(r, 0) \text {, }
$$$$
\frac{\partial \tilde{c}_{s}^{-}}{\partial t}(r, t)=D_{s}^{-}\left[\frac{2}{r} \frac{\partial \tilde{c}_{s}^{-}}{\partial r}(r, t)+\frac{\partial^{2} \tilde{c}_{s}^{-}}{\partial r^{2}}(r, t)\right]-\bar{p}^{-}(r) \tilde{c}_{s s}^{+}(t) \text {, }
$$

$$
\begin{aligned}
\frac{\partial \tilde{c}_{s}^{-}}{\partial r}(0, t) & =0, \quad \frac{\partial \tilde{c}_{s}^{-}}{\partial r}\left(R_{s}^{-}, t\right)=-\bar{p}_{0}^{-} \tilde{c}_{s s}^{+}(t), \\
\tilde{c}_{s s}^{-}(r, 0) & =c_{s s}^{-}(r, 0)-\hat{c}_{s s}^{-}(r, 0) .
\end{aligned}
$$

To derive the electrolyte estimation error system, $\tilde{c}_{e}^{j}=c_{e}^{j}-\hat{c}_{e}^{j}$, $j \in\{-$, sep, +$\}$, we assume:

- [A9]: The electrolyte diffusivity is approximately constant in $c_{e}$, i.e. $\bar{D}_{e} \approx D_{e}\left(c_{e}\right)$.
Under [A9], the electrolyte estimation error subsystem is

$$
\begin{aligned}
\frac{\partial \tilde{c}_{e}^{-}}{\partial t}(x, t) & =\bar{D}_{e} \frac{\partial^{2} \tilde{c}_{e}^{-}}{\partial x^{2}}(x, t), \\
\frac{\partial \tilde{c}_{e}^{\text {sep }}}{\partial t}(x, t) & =\bar{D}_{e} \frac{\partial^{2} \tilde{c}_{e}^{\text {sep }}}{\partial x^{2}}(x, t), \\
\frac{\partial \tilde{c}_{e}^{+}}{\partial t}(x, t) & =\bar{D}_{e} \frac{\partial^{2} \tilde{c}_{e}^{+}}{\partial x^{2}}(x, t),
\end{aligned}
$$

with boundary conditions

$$
\begin{aligned}
& \frac{\partial \tilde{c}_{e}^{-}}{\partial x}\left(0^{-}, t\right)=\frac{\partial \tilde{c}_{e}^{+}}{\partial x}\left(0^{+}, t\right)=0, \\
& \varepsilon_{e}^{-} \bar{D}_{e} \frac{\partial \tilde{c}_{e}^{-}}{\partial x}\left(L^{-}, t\right)=\varepsilon_{e}^{\mathrm{sep}} \bar{D}_{e} \frac{\partial \tilde{c}_{e}^{\mathrm{sep}}}{\partial x}\left(0^{\mathrm{sep}}, t\right), \\
& \varepsilon_{e}^{\mathrm{sep}} \bar{D}_{e} \frac{\partial \tilde{c}_{e}^{\mathrm{sep}}}{\partial x}\left(L^{\mathrm{sep}}, t\right)=\varepsilon_{e}^{+} \bar{D}_{e} \frac{\partial \tilde{c}_{e}^{+}}{\partial x}\left(L^{+}, t\right), \\
& \tilde{c}_{e}^{-}\left(L^{-}, t\right)=\tilde{c}_{e}^{\mathrm{sep}}\left(0^{\mathrm{sep}}, t\right), \\
& \tilde{c}_{e}^{\mathrm{sep}}\left(L^{\mathrm{sep}}, t\right)=\tilde{c}_{e}^{+}\left(L^{+}, t\right),
\end{aligned}
$$

and initial condition

$$
\tilde{c}_{e}^{j}(x, 0)=c_{e}^{j}(x, 0)-\hat{c}_{e}^{j}(x, 0), \quad j \in\{-, \operatorname{sep},+\} .
$$

We are now positioned to state stability of the combined estimation error systems.

Theorem 1. Consider the combined estimation error dynamics (79)-(93) for $\tilde{c}_{s}^{+}(r, t), \tilde{c}_{s}^{-}(r, t), \tilde{c}_{e}^{j}(x, t)$.

1) The solid phase estimation error subsystem (79)-(84) converges asymptotically to the zero equilibrium if $\hat{c}_{s}^{ \pm}(r, 0)$ verifies (63). That is $\lim _{t \rightarrow \infty} \tilde{c}_{s}^{+}(r, t)=$ $\lim _{t \rightarrow \infty} \tilde{c}_{s}^{-}(r, t)=0$, uniformly in $r$.

2) The electrolyte phase estimation error system (85)(93) converges asymptotically to the zero equilibrium if $\hat{c}_{e}^{j}(x, 0)$ verifies $(73)$.

Proof: The solid phase error subsystem $\tilde{c}_{s}^{ \pm}$and electrolyte phase error subsystem $\tilde{c}_{e}^{j}$ are decoupled. Therefore we can prove statements 1 and 2 separately.

Solid Phase Error System: Note the error subsystems form a cascade. Upstream subsystem $\tilde{c}_{s}^{+}$is autonomous and downstream subsystem $\tilde{c}_{s}^{-}$evolves with $\tilde{c}_{s s}^{+}(t)$ as an input. The upstream subsystem $\tilde{c}_{s}^{+}$is exponentially stable by construction of the backstepping observer (Theorem 2 of [32]). Consequently, we focus on stability of downstream subsystem $\tilde{c}_{s}^{-}$with zero input, i.e. $\tilde{c}_{s s}^{+}(t)=0$,

$$
\begin{aligned}
& \frac{\partial \tilde{c}_{s}^{-}}{\partial t}(r, t)=D_{s}^{-}\left[\frac{2}{r} \frac{\partial \tilde{c}_{s}^{-}}{\partial r}(r, t)+\frac{\partial^{2} \tilde{c}_{s}^{-}}{\partial r^{2}}(r, t)\right], \\
& \frac{\partial \tilde{c}_{s}^{-}}{\partial r}(0, t)=\frac{\partial \tilde{c}_{s}^{-}}{\partial r}\left(R_{s}^{-}, t\right)=0 .
\end{aligned}
$$

It is straightforward to show this system has infinite equilibria, since it contains one eigenvalue at the origin and the remaining eigenvalues exist on the negative real axis. Consequently, the anode error dynamics are marginally stable.

Not coincidentally, the structure and initialization of the observer ensure the anode error system converges asymptotically to the zero equilibrium. Consider the error between the total moles of lithium in the plant and observer

$$
n_{L i, s}(t)-\hat{n}_{L i, s}(t)=n_{L i, s}-n_{L i, s}=0 \quad \forall t \in \mathbb{R}^{+},
$$


by assumption [A7], Proposition 2, and construction of the estimator initial condition. Rewriting the equation above in terms of the error states,

$$
0=\sum_{j \in\{+,-\}} \frac{\varepsilon_{s}^{j} L^{j}}{\frac{4}{3} \pi\left(R_{s}^{j}\right)^{3}} \int_{0}^{R_{s}^{j}} 4 \pi r^{2} \tilde{c}_{s}^{j}(r, t) d r, \quad \forall t \in \mathbb{R}^{+} .
$$

Consider the limit as time approaches infinity

$$
\begin{aligned}
& 0=\lim _{t \rightarrow \infty}\left\{\sum_{j \in\{+,-\}} \frac{\varepsilon_{s}^{j} L^{j}}{\frac{4}{3} \pi\left(R_{s}^{j}\right)^{3}} \int_{0}^{R_{s}^{j}} 4 \pi r^{2} \tilde{c}_{s}^{j}(r, t) d r\right\}, \\
& 0=\sum_{j \in\{+,-\}} \frac{\varepsilon_{s}^{j} L^{j}}{\frac{4}{3} \pi\left(R_{s}^{j}\right)^{3}} \int_{0}^{R_{s}^{j}} 4 \pi r^{2} \cdot \lim _{t \rightarrow \infty} \tilde{c}_{s}^{j}(r, t) d r .
\end{aligned}
$$

Now apply the following two properties,

1) $\lim _{t \rightarrow \infty} \tilde{c}_{s}^{+}(r, t)=0$ uniformly in $r$, due to the exponential stability of (79)-(80) [32].

2) The equilibrium structure of (94)-(95) implies $\lim _{t \rightarrow \infty} \tilde{c}_{s}^{-}(r, t)=\tilde{c}_{s, e q}^{-}$is uniform in $r \in\left[0, R_{s}^{-}\right]$

Then (98) reduces to

$$
\begin{aligned}
& 0=\frac{\varepsilon_{s}^{-} L^{-}}{\frac{4}{3} \pi\left(R_{s}^{-}\right)^{3}} \int_{0}^{R_{s}^{-}} 4 \pi r^{2} \cdot \tilde{c}_{s, e q}^{-} d r, \\
& 0=\varepsilon_{s}^{-} L^{-} \cdot \tilde{c}_{s, e q}^{-}, \\
& 0=\tilde{c}_{s, e q}^{-} .
\end{aligned}
$$

Therefore the subsystem (94)-(95) converges asymptotically to the zero equilibrium, that is $\lim _{t \rightarrow \infty} \tilde{c}_{s}^{-}(r, t)=0 \forall r \in$ $\left[0, R_{s}^{-}\right]$. Since the error subsystems form a cascade, the composite error system is asymptotically stable.

Electrolyte Phase Error System: The proof of statement 2 consists of three main steps. First, apply coordinate scaling and composition to transform (85)-(93) into a heat equation with homogeneous Neumann boundary conditions. Second, compute the solution using separation of variables. Third, show the solution converges asymptotically to the zero equilibrium if (73) is verified.

Step 1: For notational simplicity, denote $\delta_{1}=\varepsilon_{e}^{-} \bar{D}_{e}, \delta_{2}=$ $\varepsilon_{e}^{\mathrm{sep}} \bar{D}_{e}, \delta_{3}=\varepsilon_{e}^{+} \bar{D}_{e}$. Next, decompose and transform the $x$ coordinate as follows,

$$
\begin{aligned}
& \xi_{1}=\frac{x}{\delta_{1}}, \quad \xi_{2}=\frac{x}{\delta_{2}}+\left(\frac{L^{-}}{\delta_{1}}-\frac{L^{-}}{\delta_{2}}\right), \\
& \xi_{3}=\frac{x}{\delta_{3}}+\left(\frac{L^{\mathrm{sep}}-L^{-}}{\delta_{2}}+\frac{L^{-}}{\delta_{1}}-\frac{L^{\mathrm{sep}}}{\delta_{3}}\right) .
\end{aligned}
$$

which yields

$$
\begin{aligned}
\frac{\partial \tilde{c}_{e}^{-}}{\partial t}\left(\xi_{1}, t\right) & =\frac{\bar{D}_{e}}{\delta_{1}^{2}} \frac{\partial^{2} \tilde{c}_{e}^{-}}{\partial \xi_{1}^{2}}\left(\xi_{1}, t\right), \\
\frac{\partial \tilde{c}_{e}^{\text {sep }}}{\partial t}\left(\xi_{2}, t\right) & =\frac{\bar{D}_{e}}{\delta_{2}^{2}} \frac{\partial^{2} \tilde{c}_{e}^{\text {sep }}}{\partial \xi_{2}^{2}}\left(\xi_{2}, t\right), \\
\frac{\partial \tilde{c}_{e}^{+}}{\partial t}\left(\xi_{3}, t\right) & =\frac{\bar{D}_{e}}{\delta_{3}^{2}} \frac{\partial^{2} \tilde{c}_{e}^{+}}{\partial \xi_{3}^{2}}\left(\xi_{3}, t\right),
\end{aligned}
$$

with boundary conditions

$$
\frac{\partial \tilde{c}_{e}^{-}}{\partial \xi_{1}}\left(l_{01}, t\right)=\frac{\partial \tilde{c}_{e}^{+}}{\partial \xi_{3}}\left(l_{30}, t\right)=0
$$

$$
\begin{aligned}
& \frac{\partial \tilde{c}_{e}^{-}}{\partial \xi_{1}}\left(l_{12}, t\right)=\frac{\partial \tilde{c}_{e}^{\text {sep }}}{\partial \xi_{2}}\left(l_{12}, t\right), \\
& \frac{\partial \tilde{c}_{e}^{\text {sep }}}{\partial \xi_{2}}\left(l_{23}, t\right)=\frac{\partial \tilde{c}_{e}^{+}}{\partial \xi_{3}}\left(l_{23}, t\right), \\
& \tilde{c}_{e}^{-}\left(l_{12}, t\right)=\tilde{c}_{e}^{\text {sep }}\left(l_{12}, t\right), \\
& \tilde{c}_{e}^{\text {sep }}\left(l_{23}, t\right)=\tilde{c}_{e}^{+}\left(l_{23}, t\right),
\end{aligned}
$$

where

$$
\begin{aligned}
& l_{01}=\frac{0^{-}}{\delta_{1}}, \quad l_{12}=\frac{L^{-}}{\delta_{1}}, \quad l_{23}=\frac{L^{-}}{\delta_{1}}+\frac{L^{\mathrm{sep}}-L^{-}}{\delta_{2}}, \\
& l_{30}=\frac{L^{-}}{\delta_{1}}+\frac{L^{\mathrm{sep}}-L^{-}}{\delta_{2}}+\frac{0^{+}-L^{\mathrm{sep}}}{\delta_{3}} .
\end{aligned}
$$

We now concatenate the spatial coordinate, state, and diffusion coefficient into single variables,

$$
\begin{gathered}
\xi= \begin{cases}\xi_{1} & \text { if } l_{01} \leq \xi \leq l_{12} \\
\xi_{2} & \text { if } l_{12}<\xi<l_{23} \\
\xi_{3} & \text { if } l_{23} \leq \xi \leq l_{30},\end{cases} \\
w(\xi, t)= \begin{cases}\tilde{c}_{e}^{-}(\xi, t) & \text { if } l_{01} \leq \xi \leq l_{12} \\
\tilde{c}_{e}^{\mathrm{sep}}(\xi, t) & \text { if } l_{12}<\xi<l_{23} \\
\tilde{c}_{e}^{+}(\xi, t) & \text { if } l_{23} \leq \xi \leq l_{30},\end{cases} \\
\bar{D}(\xi)= \begin{cases}\frac{\bar{D}_{e}}{\delta_{1}^{2}} & \text { if } l_{01} \leq \xi \leq l_{12} \\
\frac{\bar{D}_{e}}{\delta_{2}^{2}} & \text { if } l_{12}<\xi<l_{23} \\
\frac{\bar{D}_{e}}{\delta_{3}^{2}} & \text { if } l_{23} \leq \xi \leq l_{30} .\end{cases}
\end{gathered}
$$

By continuity of $\xi$ and $\tilde{c}_{e}^{j}(\xi, t)$ across the three regions in (103)-(110), we can re-write the system into a simple heat equation with homogeneous Neumann boundary conditions,

$$
\begin{aligned}
\frac{\partial w}{\partial t}(\xi, t) & =\bar{D}(\xi) \frac{\partial^{2} w}{\partial \xi^{2}}(\xi, t), \\
\frac{\partial w}{\partial \xi}\left(l_{01}, t\right) & =\frac{\partial w}{\partial \xi}\left(l_{30}, t\right)=0, \\
w(\xi, 0) & =c_{e}(\xi, 0)-\hat{c}_{e}(\xi, 0) .
\end{aligned}
$$

Next we normalize the spatial variable $\zeta=\left(\xi-l_{01}\right) /\left(l_{30}-l_{01}\right)$ to obtain

$$
\begin{aligned}
\frac{\partial w}{\partial t}(\zeta, t) & =\overline{\bar{D}}(\zeta) \frac{\partial^{2} w}{\partial \zeta^{2}}(\zeta, t), \\
\frac{\partial w}{\partial \zeta}(0, t) & =\frac{\partial w}{\partial \xi}(1, t)=0, \\
w(\zeta, 0) & =c_{e}(\zeta, 0)-\hat{c}_{e}(\zeta, 0) .
\end{aligned}
$$

where $\overline{\bar{D}}(\zeta)=\frac{1}{\left(l_{30}-l_{01}\right)^{2}} \bar{D}\left(\left(l_{30}-l_{01}\right) \zeta+l_{01}\right)$. The final step is to apply a "gauge" transformation [37] to obtain a heat equation with a spatially invariant diffusion coefficient,

$$
z=\sqrt{D_{0}} \int_{0}^{\zeta} \frac{d s}{\sqrt{\overline{\bar{D}}(s)}}, \quad \text { where } D_{0}=\left(\int_{0}^{1} \frac{d s}{\sqrt{\overline{\bar{D}}(s)}}\right)^{-2}
$$

This yields the heat equation

$$
\begin{aligned}
& \frac{\partial w}{\partial t}(z, t)=D_{0} \frac{\partial^{2} w}{\partial z^{2}}(z, t), \\
& \frac{\partial w}{\partial z}(0, t)=\frac{\partial w}{\partial z}(1, t)=0,
\end{aligned}
$$




$$
w(z, 0)=c_{e}(z, 0)-\hat{c}_{e}(z, 0) .
$$

Step 2: Next we solve (122)-(124) using separation of variables. Let us consider the solution form:

$$
w(z, t)=Z(z) \cdot T(t) .
$$

Following the procedure in many PDE textbooks (see e.g. [37], [38]), we obtain the solution,

$$
w(z, t)=A_{0}+\sum_{n=1}^{\infty} A_{n} \cos (n \pi z) \cdot e^{-D_{0} \cdot n^{2} \pi^{2} t},
$$

where the Fourier Sine Series coefficients are

$$
\begin{aligned}
& A_{0}=\int_{0}^{1} w(z, 0) d z \\
& A_{n}=2 \int_{0}^{1} w(z, 0) \cos (n \pi z) d z, \quad n=1,2,3, \cdots .
\end{aligned}
$$

Note the second term on the right hand side of (126) decays to zero exponentially fast in time. Therefore, we focus on showing $A_{0}=0$.

Step 3: Next we show $A_{0}=0$ in (127). To prove this, we perform the reverse coordinate transformation procedure from Step 1 , namely $z \mapsto \zeta \mapsto \xi \mapsto\left(\xi_{1}, \xi_{2}, \xi_{3}\right) \mapsto x$. This ultimately results in,

$A_{0}=\int_{0}^{1} w(z, 0) d z=\sqrt{D_{0} \bar{D}_{e}} \sum_{j \in\{-, \mathrm{sep},+\}} \varepsilon_{e}^{j} \int_{0^{j}}^{L^{j}} \tilde{c}_{e}^{j}(x, 0) d x$

Using the definition of $n_{L i, e}$ in (35) and applying initial condition constraint (73) yields

$$
A_{0}=\int_{0}^{1} w(z, 0) d z=\sqrt{D_{0} \bar{D}_{e}}\left(n_{L i, e}-n_{L i, e}\right)=0 .
$$

As a result, $w(z, t)$ converges asymptotically (exponentially, in fact) to zero. Since all transformations are invertible, (79)-(83) converges asymptotically to the zero equilibrium.

Remark 4. The solution for $w(x, t)$ in (126) exposes the dynamical structure for the electrolyte's estimation error dynamics. Namely, the solution is characterized by a constant $A_{0}$ determined by the error in the initial estimation of the total amount of lithium in the electrolyte, plus exponentially decaying terms with eigenvalues $\lambda_{n}=-D_{0} \cdot n^{2} \pi^{2}$ on the negative real axis of the complex plane that increase quadratically toward $-\infty$ as $n$ increases.

\section{B. Output Function Inversion Convergence}

In the following analysis, we prove convergence of the output inversion scheme over a compact set of surface concentration, which satisfies the following assumption:

- [A10]: The output function (74) has negative and bounded gradient:

$-M_{2} \leq \frac{\partial h}{\partial c_{s s}^{+}}\left(c_{s s}^{+}, t\right) \leq-M_{1}<0, \quad \forall c_{s s}^{+} \in \mathcal{D}, t \in \mathbb{R}^{+}$,

where $0<M_{1}<M_{2}$. For example, we can pick $\mathcal{D}=$ $\left[0.1 c_{s, \max }^{+}, 0.8 c_{s, \max }^{+}\right]$from Fig. 6.
Theorem 2. Let the error between the true and processed values be $\tilde{\theta}=c_{s s}^{+}-\check{c}_{s s}^{+}$. Furthermore, assume the dynamics of $\check{c}_{s s}^{+}$are significantly faster than $c_{s s}^{+}$. Then $\tilde{\theta}(t)$ evolves according to the error system,

$$
\frac{d}{d t} \tilde{\theta}(t)=-\gamma \phi e_{n l}
$$

where

$$
\gamma>0, \quad \phi=\frac{\partial h}{\partial c_{s s}^{+}}\left(\check{c}_{s s}^{+}, t\right), \quad e_{n l}=h\left(c_{s s}^{+}, t\right)-h\left(\check{c}_{s s}^{+}, t\right) .
$$

Under [A10], $\tilde{\theta}$ converges exponentially to the zero equilibrium on the compact set $c_{s s}^{+}, \check{c}_{s s}^{+} \in \mathcal{D}$. More specifically, for all initial conditions $c_{s s}^{+}(0), \check{c}_{s s}^{+}(0) \in \mathcal{D}$, the following holds:

$$
|\tilde{\theta}(0)|^{2} \cdot e^{-2 \gamma \cdot M_{2}^{2} \cdot t} \leq|\tilde{\theta}(t)|^{2} \leq|\tilde{\theta}(0)|^{2} \cdot e^{-2 \gamma \cdot M_{1}^{2} \cdot t}, \quad \forall t \in R^{+},
$$

Proof: The proof uses the Mean Value Theorem approach for systems with monotone nonlinearities [39], [40]. Consider

$$
e_{n l}=h\left(c_{s s}^{+}, t\right)-h\left(\check{c}_{s s}^{+}, t\right)=\chi(1)-\chi(0)
$$

where we define $\chi(\alpha)=h\left(\check{c}_{s s}^{+}+\alpha \tilde{\theta}, t\right)$. The total derivative of $\chi(\alpha)$ is

$$
\frac{d \chi}{d \alpha}(\alpha)=\frac{\partial h}{\partial c_{s s}^{+}} \frac{d c_{s s}^{+}}{d \alpha}=\frac{\partial h}{\partial c_{s s}^{+}} \tilde{\theta} .
$$

Now we invoke the fundamental theorem of calculus

$$
e_{n l}=\chi(1)-\chi(0)=\int_{0}^{1} \frac{d \chi}{d \alpha}(\alpha) d \alpha=\int_{0}^{1} \frac{\partial h}{\partial c_{s s}^{+}} \cdot \tilde{\theta} \cdot d \alpha .
$$

We are now positioned to propose Lyapunov function,

$$
W(t)=\frac{1}{2 \gamma} \tilde{\theta}^{2}(t)
$$

The derivative of $W(t)$ along the trajectories of $\tilde{\theta}$ is given by

$$
\begin{aligned}
\frac{d}{d t} W(t) & =\frac{1}{\gamma} \tilde{\theta}(t) \dot{\tilde{\theta}}(t)=-\tilde{\theta} \cdot \phi \cdot e_{n l}, \\
& =-\tilde{\theta} \cdot \frac{\partial h}{\partial c_{s s}^{+}} \cdot \int_{0}^{1} \frac{\partial h}{\partial c_{s s}^{+}} \cdot \tilde{\theta} \cdot d \alpha,
\end{aligned}
$$

where we have used (132), (133), (137). Applying (131) from [A10] yields

$$
\begin{aligned}
-M_{2}^{2} \cdot \tilde{\theta}^{2}(t) & \leq \frac{d}{d t} W(t) \leq-M_{1}^{2} \cdot \tilde{\theta}^{2}(t), \\
-M_{2}^{2} \cdot 2 \gamma \cdot W(t) & \leq \frac{d}{d t} W(t) \leq-M_{1}^{2} \cdot 2 \gamma \cdot W(t) .
\end{aligned}
$$

Therefore, we conclude the zero equilibrium of (132) is exponentially stable [41]. Applying the comparison principle yields

$$
W(0) \cdot e^{-2 \gamma \cdot M_{2}^{2} \cdot t} \leq W(t) \leq W(0) \cdot e^{-2 \gamma \cdot M_{1}^{2} \cdot t}, \quad \forall t \in R^{+} .
$$

Using the definition of $W(t)$ in (138) yields

$$
|\tilde{\theta}(0)|^{2} \cdot e^{-2 \gamma \cdot M_{2}^{2} \cdot t} \leq|\tilde{\theta}(t)|^{2} \leq|\tilde{\theta}(0)|^{2} \cdot e^{-2 \gamma \cdot M_{1}^{2} \cdot t}, \quad \forall t \in R^{+} .
$$

Remark 5 (Output Inversion Limitation). The significance of assumption [A10] and Thm 2 is that convergence is guaranteed 
only when $h\left(c_{s s}^{+}, t\right)$ is strictly decreasing w.r.t. $c_{s s}^{+}$. If the gradient $\partial h / \partial c_{s s}^{+}$is zero, then convergence is not guaranteed, a property we explore by simulation in Section V.A.

Remark 6 (Stability Analysis for Fully Composed Observer). The preceding subsections analyze convergence of the individual observer systems (solid + electrolyte phase, output inversion), assuming perfect knowledge of the corresponding inputs. Analyzing stability of the fully composed observer is very complex, given the interconnected structure of the observer subsystems. Specifically, the solid phase observer and output inversion are interconnected, whereas the electrolyte observer is autonomous. Instead, we pursue simulations next to obtain empirical insight into the fully composed observer's convergence properties.

\section{Simulations}

This section presents simulations that demonstrate the SPMe observer's performance. Ideally, we prefer experimentally measured electrochemical state data to validate the proposed observer. Unfortunately, in situ measurements of lithium concentrations are very difficult. The few successful efforts use neutron imaging on custom-made and test-specific cells, such as [42], [43], [44]. In this manuscript, we apply the observer to the DFN model (1)-(21). The model parameters used in this study originate from the publicly available DUALFOIL simulation package [30] and correspond to a $\mathrm{LiCoO}_{2}$ cathode / graphite anode chemistry. The DFN model's numerical implementation is summarized in Section II.C of [1].

For all presented simulations the state estimates are initialized at incorrect values. Namely, the true initial condition is $c_{s}^{-}(r, 0) / c_{s, \max }^{-}=0.8224$ and the observer's initial condition is $\hat{c}_{s}^{-}(r, 0) / c_{s, \max }^{-}=0.4$. The following important parameters are also assumed: $n_{L i, s}=2.5$ moles, $n_{L i, e}=0.085$ moles, $\lambda=-0.5, \gamma=10^{8}$, unless otherwise specified. Surface concentrations are given by $\theta^{ \pm}=c_{s s}^{ \pm} / c_{s, \text { max }}^{ \pm}$.

Ultimately, the PDE-based observer must be discretized into ODEs for simulation. Although numerical schemes are not the focus of this paper (see e.g. [45], [46] and references therein), we summarize the methods applied here. The solid phase and electrolyte observers are discretized in space via the central finite difference method, with 2nd-order one-sided finite differences at all boundary conditions [47]. A crucial step in the numerical integration scheme is to supply analytic Jacobians, which accelerates convergence and increases accuracy [1].

\section{A. Constant 1C Discharge Cycle}

First we consider a constant $1 \mathrm{C}$ discharge cycle in Fig. 8. Sustained C-rates are challenging since they induce nonnegligible electrolyte gradients. Despite incorrect initial conditions and model mismatch (SPMe observer vs DFN truth model), the processed cathode surface concentration $\check{\theta}^{+}(t)$ converges to the true value, followed by convergent estimates of surface concentrations $\hat{\theta}^{-}(t), \hat{\theta}^{+}(t)$. After the initial transient $(\sim 750 \mathrm{sec})$, the estimates have RMS errors of $2.39 \%$, $1.46 \%$, and $8.6 \mathrm{mV}$ for the anode surface concentration, cathode surface concentration, and voltage, respectively. Note that

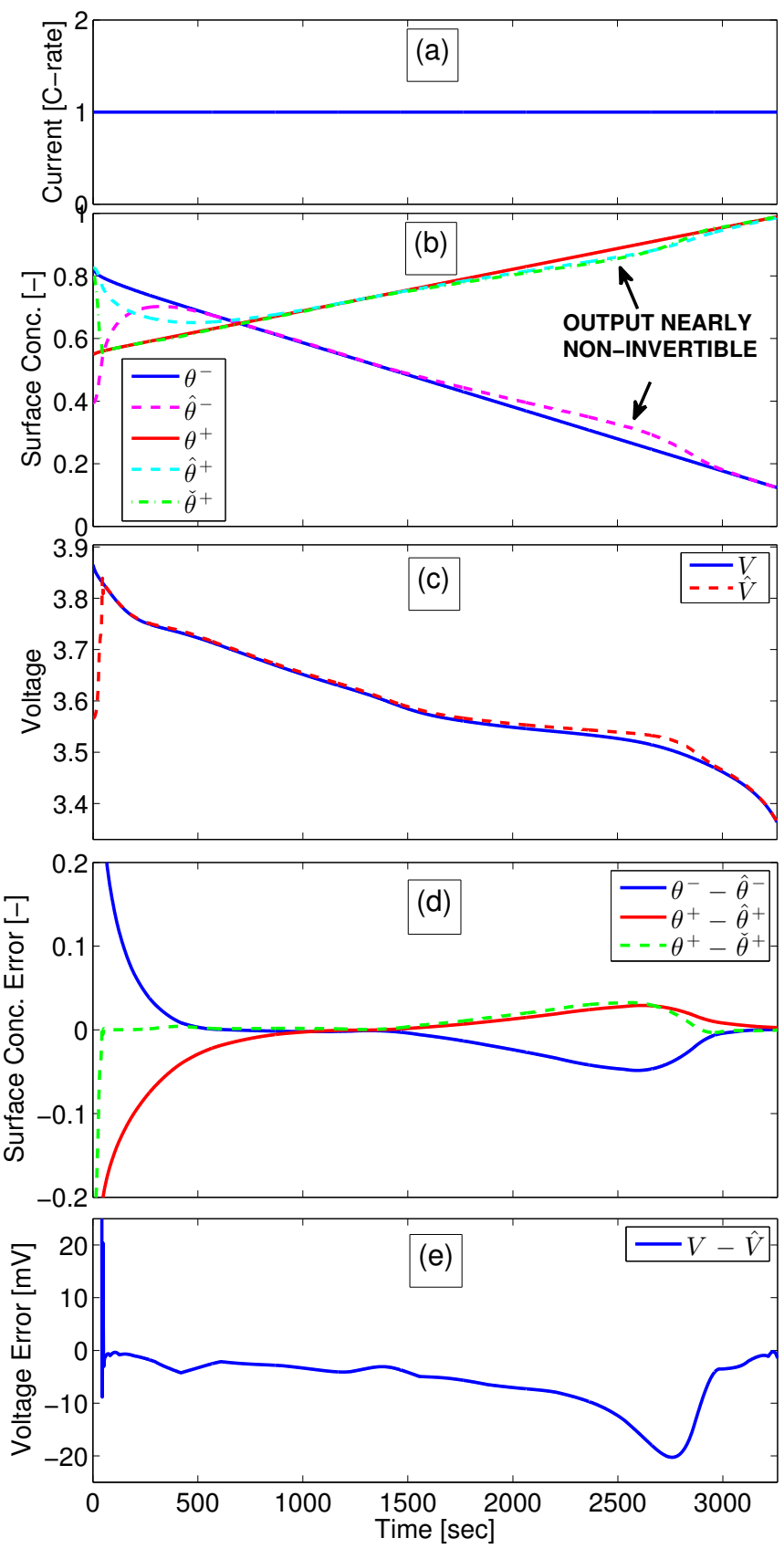

Fig. 8. SPMe observer results for a $1 \mathrm{C}$ constant current discharge, using DFN as truth model. True initial condition: $c_{s}^{-}(r, 0) / c_{s, \max }^{-}=0.8224$. Observer initial condition: $\hat{c}_{s}^{-}(r, 0) / c_{s, \max }^{-}=0.4$. (a) input current; (b) surface concentrations $\theta^{ \pm}=c_{s s}^{ \pm} / c_{s, \text { max }}^{ \pm}$; (c) voltage; (d) surface concentration estimation error; (e) voltage estimation error.

the estimates temporarily diverge in the range $0.8<\theta^{+}<0.9$. In this range, the output function's derivative w.r.t. $c_{s s}^{+}$is nearly zero (see Fig. 6), meaning the output function is nearly noninvertible. This feature is a fundamental limitation of battery state estimation, as discussed previously in the literature [29] and exposed in Thm 2. The estimates recover as $\theta^{+}$enters a more strongly invertible range. Note the SPMe observer resolves the constant high C-rate deficiencies of previous SPM-based observers (e.g. Section 7.2 of [15]). 


\section{B. Electric Vehicle Charge/Discharge Cycle}

Second we apply an electric vehicle charge/discharge cycle in Fig. 9. This input signal in Fig. 9(a) is generated from two concatenated UDDS drive cycles simulated on models developed in [31]. Unlike the previous input, this cycle is characterized by larger non-sustained C-rates. The mean square Crate is $1.28 \mathrm{C}$ and the absolute maximum $\mathrm{C}$-rate is $5.43 \mathrm{C}$. After the initial transient $(\sim 750 \mathrm{sec})$, the estimates have RMS errors of $0.60 \%, 0.30 \%$, and $6.7 \mathrm{mV}$ for the anode surface concentration, cathode surface concentration, and voltage, respectively (see Fig. 9(d)-(e)). Note the SPMe observer estimates output voltage with significantly higher accuracy than previous SPMbased observers (e.g. Section 7.1 of [15]). Similar estimation results occur for various other initial conditions and drive cycle inputs, including US06, SC04, LA92, and naturalistic micro trip data.

\section{Error in Moles of Solid Lithium $n_{L i, s}$}

Third, we examine robustness with respect to error in the perceived moles of solid lithium, i.e. $\hat{n}_{L i, s}$. Recall from Section III-E that a correct value of $n_{L i, s}$ is required for stability [A7]. We now relax this assumption. This elucidates the impact of capacity fade on the observer performance. Again, we apply the UDDS charge/discharge cycle in Fig. 9(a), but intentionally supply the observer with $5 \%$ more lithium than the truth model, i.e. $\hat{n}_{L i, s}=1.05 \times n_{L i, s}=2.625$ moles. The simulation reported in Fig. 10 demonstrates an estimation bias resulting from an inaccurate total amount of solid lithium. After the initial transient ( $750 \mathrm{sec})$, the RMS estimation errors are $5.20 \%$ and $2.87 \%$ for the anode and cathode surface concentrations, respectively. This is roughly a 10x error increase relative to the case with a correct $n_{L i, s}$ value. Importantly, note the voltage estimation error $(7.4 \mathrm{mV}$ RMS) is nearly the same as with a correct value of $n_{L i, s}$, despite biased concentration estimates. This occurs because the observer converges on overestimated surface concentrations that satisfy $\hat{n}_{L i, s}=1.05 \times n_{L i, s}$ and produce a solid potential difference that yields the same value as the true concentrations. Therefore, the SPMe observer achieves estimated output convergence, yet produces biased solid concentration state estimates. This motivates (i) accurate knowledge of $n_{L i, s}$ for a fresh cell, and (ii) a real-time parameter estimation algorithm to determine how $n_{L i, s}$ evolves as the battery ages [15].

\section{Error in Moles of Electrolyte Lithium $n_{L i, e}$}

Finally, we examine robustness with respect to error in the perceived moles of electrolyte lithium, i.e. $\hat{e}_{L i, s}$. Recall from Section III-F that a correct value of $n_{L i, e}$ is required for stability [A8]. We now investigate the impact of relaxing this assumption. Again, we apply the UDDS charge/discharge cycle in Fig. 9(a), but intentionally supply the observer with $5 \%$ more lithium than the truth model, i.e. $\hat{n}_{L i, e}=1.05 \times n_{L i, e}$ $=0.0893$ moles. Interestingly, the simulation reported in Fig. 11(a),(c) demonstrates no estimation bias for the surface concentrations. Namely, the RMS estimation errors after the initial transient $(\sim 750 \mathrm{sec})$ are $0.58 \%$ and $0.33 \%$ for the anode and
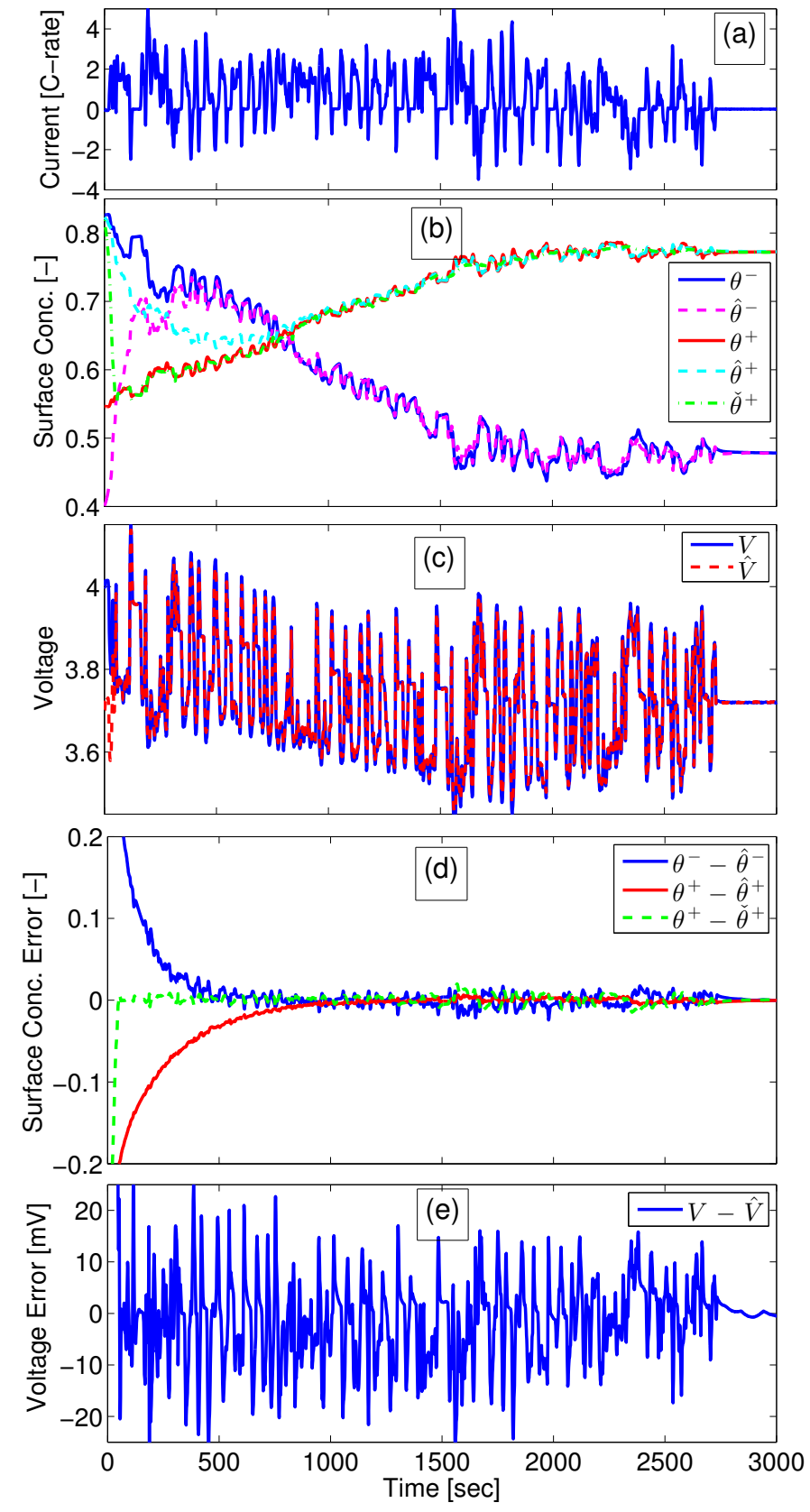

Fig. 9. SPMe observer results for a UDDSx2 charge/discharge cycle, using DFN as truth model. True initial condition: $c_{s}^{-}(r, 0) / c_{s, \max }^{-}=0.8224$. Observer initial condition: $\hat{c}_{s}^{-}(r, 0) / c_{s, \max }^{-}=0.4$. (a) input current; (b) surface concentrations $\theta^{ \pm}=c_{s s}^{ \pm} / c_{s, \text { max }}^{ \pm}$; (c) voltage; (d) surface concentration estimation error; (e) voltage estimation error.

cathode surface concentrations, respectively. This is similar to the results with a correct value of $n_{L i, e}$. Moreover, the voltage estimation error remains small in Fig. 11(e), $7.5 \mathrm{mV}$ RMS. However, there is clear bias in the electrolyte concentration estimate. Figure 11(b) plots the electrolyte concentrations at the current collectors, $c_{e}^{ \pm}\left(0^{ \pm}, t\right)$ and the estimation error $c_{e}^{ \pm}\left(0^{ \pm}, t\right)-\hat{c}_{e}^{ \pm}\left(0^{ \pm}, t\right)$ is in Fig. 11(d). The RMS errors for the anode and cathode are $7.1 \%$ and $6.6 \%$, respectively. These results can be explained by: (i) the electrolyte dynamics are decoupled from the solid-phase dynamics (c.f. Fig. 2), and 

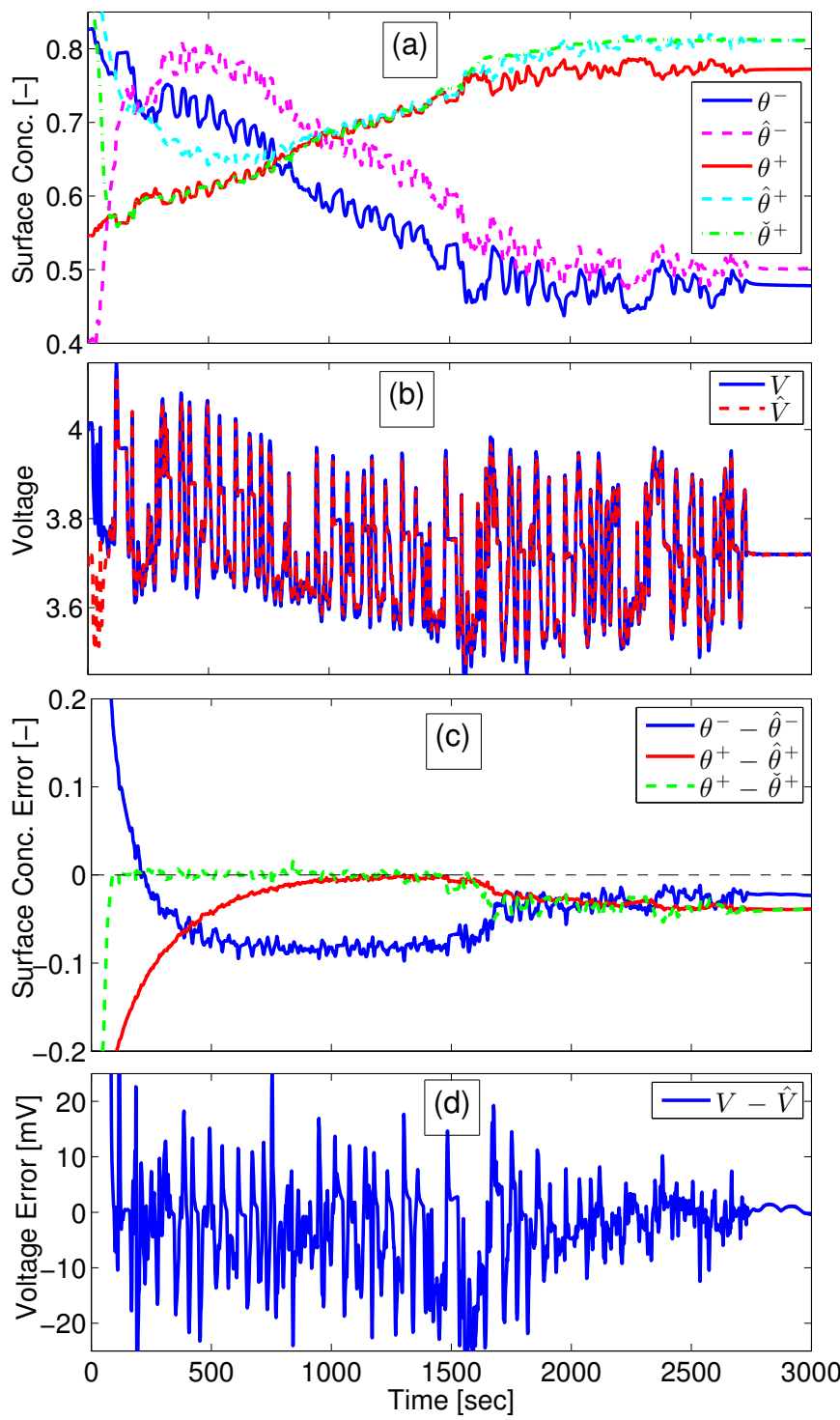

Fig. 10. SPMe observer results for a UDDSx2 charge/discharge cycle, using DFN as truth model and incorrect $n_{L i, s}$ value. True initial condition: $c_{s}^{-}(r, 0) / c_{s, \max }^{-}=0.8224$. Observer initial condition: $\hat{n}_{L i, s}=$ $1.05 \times n_{L i, s}, \hat{c}_{s}^{-}(r, 0) / c_{s, \max }^{-}=0.4$. (a) surface concentrations $\theta^{ \pm}=$ $c_{s s}^{ \pm} / c_{s, \text { max }}^{ \pm}$; (b) voltage; (c) surface concentration estimation error; (d) voltage estimation error.

(ii) the voltage output depends on the difference $\ln c_{e}\left(0^{+}, t\right)-$ $\ln c_{e}\left(0^{-}, t\right)$ (see (32)). That is, the absolute values of $c_{e}^{ \pm}\left(0^{ \pm}, t\right)$ can be incorrect, provided that the difference between their natural logarithms is correct.

Here, we have examined robustness to parametric uncertainty in $n_{L i, s}$ and $n_{L i, e}$. It is also important to consider robustness to other uncertain parameters, such as diffusivities and conductivities. Comprehensively addressing this issue is an open research topic. Some initial efforts have been made. A sensitivity-based interval observer approach has been recently proposed in [48]. Electrochemical model parameter sensitivity is also examined in [33], [34] via Fisher information.
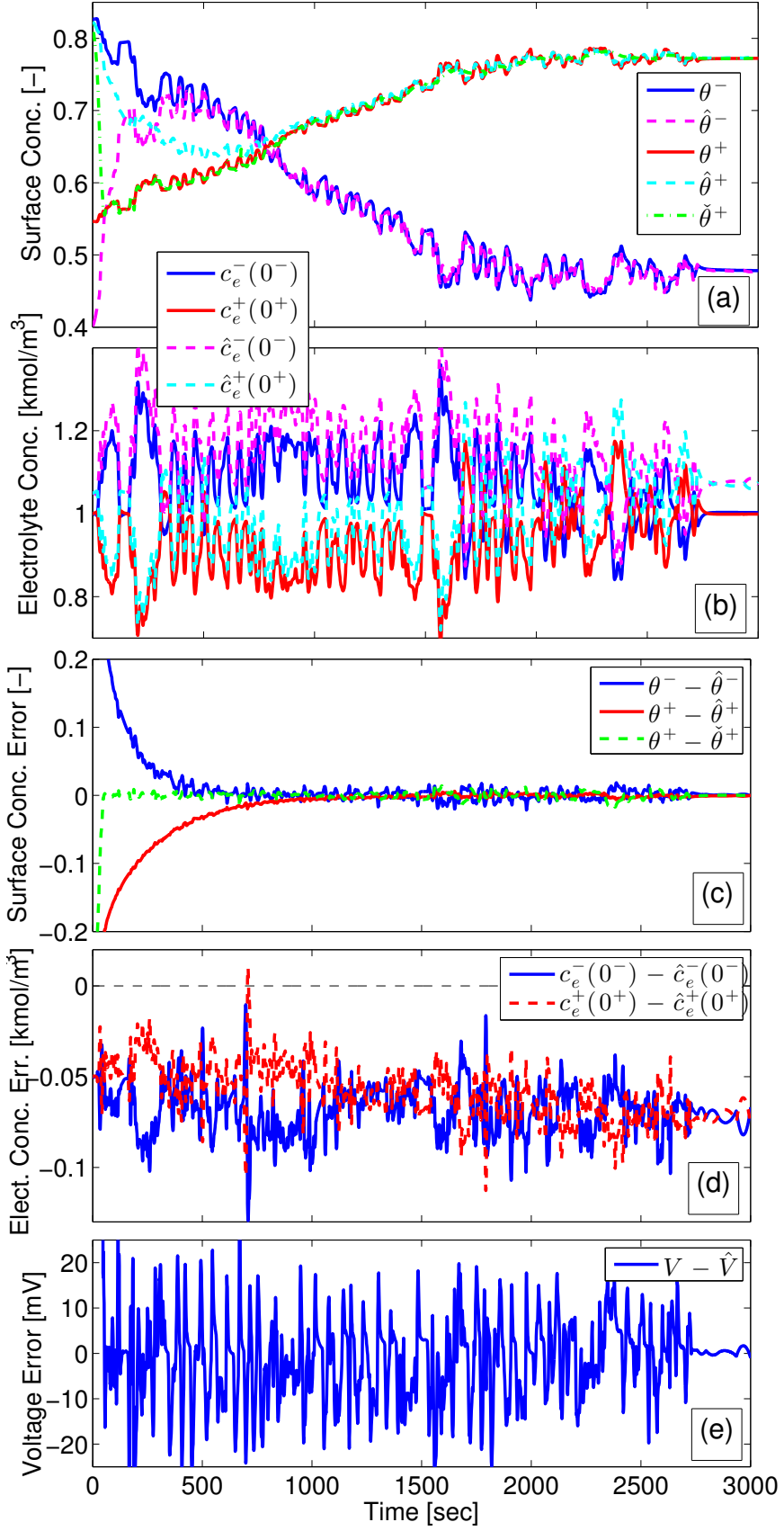

Fig. 11. SPMe observer results for a UDDSx2 charge/discharge cycle, using DFN as truth model and incorrect $n_{L i, e}$ value. True initial condition: $c_{s}^{-}(r, 0) / c_{s, \max }^{-}=0.8224$. Observer initial condition: $\hat{n}_{L i, e}=$ $1.05 \times n_{L i, e}, \hat{c}_{s}^{-}(r, 0) / c_{s, \max }^{-}=0.4$. (a) surface concentrations $\theta^{ \pm}=$ $c_{s s}^{ \pm} / c_{s, \text { max }}^{ \pm}$; (b) voltage; (c) surface concentration estimation error; (d) voltage estimation error.

\section{CONCLUSIONS}

This paper derives a reduced electrochemical battery model called the Single Particle Model with Electrolyte (SPMe). The proposed model is most similar to those developed in [18], [20], [22], yet we analyze the dynamical properties that enable a provably convergent observer design. The SPMe is compared against the Doyle-Fuller-Newman (DFN) model [2], [3] and single particle model (SPM) without electrolyte. We then examine the model's mathematical properties, including 
stability, lithium conservation, and output function invertibility. Based on several critical features of the SPMe mathematical structure, (i.e. subsystem decoupling, marginal stability, conservation of lithium, and local output invertibility), we propose a state estimation scheme summarized in Table II and prove convergence for the observer and output inversion algorithms. The proposed SPMe observer is characterized by only two tuning parameters - thereby making calibration significantly simpler than Kalman filter based estimators. Finally, we examine the performance attributes and limitations of the SPMe observer using data generated from a DFN model. Simulations demonstrate convergent estimates on high C-rate cycles and transient electric vehicle charge/discharge cycles. State estimation biases occur in the solid or electrolyte phase if the incorrect moles of lithium in the respective subsystem is assumed. This may be acceptable, depending on what states the battery control engineer wishes to accurately monitor (i.e. bulk SOC, surface concentrations, or electrolyte concentrations). Nevertheless, the bias motivates parameter identification algorithms to estimate moles of cyclable lithium in each phase - a topic for further investigation. Additionally, the proposed SPMe observer can be extended to include temperature dynamics [21] and multi-material cathodes [29] - topics for further study.

\section{REFERENCES}

[1] H. Perez, N. Shahmohammadhamedani, and S. Moura, "Enhanced performance of li-ion batteries via modified reference governors and electrochemical models," IEEE/ASME Transactions on Mechatronics, vol. 20, no. 4, pp. 1511-1520, Aug 2015.

[2] K. Thomas, J. Newman, and R. Darling, Advances in Lithium-Ion Batteries. New York, NY USA: Kluwer Academic/Plenum Publishers, 2002, ch. 12: Mathematical modeling of lithium batteries, pp. 345-392.

[3] N. A. Chaturvedi, R. Klein, J. Christensen, J. Ahmed, and A. Kojic, "Algorithms for advanced battery-management systems," IEEE Control Systems Magazine, vol. 30, no. 3, pp. 49 - 68, 2010.

[4] D. D. Domenico, A. Stefanopoulou, and G. Fiengo, "Lithium-Ion Battery State of Charge and Critical Surface Charge Estimation Using an Electrochemical Model-Based Extended Kalman Filter,' Journal of Dynamic Systems, Measurement, and Control, vol. 132, no. 6, p. 061302, 2010.

[5] X. Hu, S. Li, and H. Peng, "A comparative study of equivalent circuit models for li-ion batteries," Journal of Power Sources, vol. 198, pp. 359-367, 2012

[6] G. L. Plett, "Extended Kalman filtering for battery management systems of LiPB-based HEV battery packs. Part 3. State and parameter estimation," Journal of Power Sources, vol. 134, no. 2, pp. 277-92, 2004.

[7] Y. Hu and S. Yurkovich, "Battery cell state-of-charge estimation using linear parameter varying system techniques," Journal of Power Sources, vol. 198 , pp. $338-350,2012$.

[8] I.-S. Kim, "A technique for estimating the state of health of lithium batteries through a dual-sliding-mode observer," IEEE Transactions on Power Electronics, vol. 25, no. 4, pp. 1013 - 1022, 2010.

[9] S. Bashash and H. K. Fathy, "Battery State of Health and Charge Estimation Using Polynomial Chaos Theory," in 2013 ASME Dynamic Systems and Control Conference, Palo Alto, California, Oct 2013, p. V001T05A006.

[10] M. Charkhgard and M. Farrokhi, "State-of-charge estimation for lithiumion batteries using neural networks and ekf," IEEE Transactions on Industrial Electronics, vol. 57, no. 12, pp. 4178-4187, Dec 2010.

[11] G. L. Plett, "Sigma-point Kalman filtering for battery management systems of LiPB-based HEV battery packs. Part 2: Simultaneous state and parameter estimation," Journal of Power Sources, vol. 161, no. 2, pp. 1369-1384, 2006.

[12] H. He, R. Xiong, X. Zhang, F. Sun, and J. Fan, "State-of-charge estimation of the lithium-ion battery using an adaptive extended kalman filter based on an improved thevenin model," IEEE Transactions on Vehicular Technology, vol. 60, no. 4, pp. 1461-1469, May 2011.
TABLE III

APPENDIX: NOMENCLATURE

Symbols in order of appearance

\begin{tabular}{|c|c|}
\hline \multicolumn{2}{|r|}{ Electrochemical model states, inputs, outputs } \\
\hline$c_{s}^{ \pm}$ & Lithium concentration in solid phase $\left[\mathrm{mol} / \mathrm{m}^{3}\right]$ \\
\hline$c_{e}$ & Lithium concentration in electrolyte phase $\left[\mathrm{mol} / \mathrm{m}^{3}\right]$ \\
\hline$\phi_{s}^{ \pm}$ & Solid electric potential $[\mathrm{V}]$ \\
\hline$\phi_{e}$ & Electrolyte electric potential [V] \\
\hline$i_{e}^{ \pm}$ & Ionic current $\left[\mathrm{A} / \mathrm{m}^{2}\right]$ \\
\hline$j_{n}^{ \pm}$ & Molar ion flux $\left[\mathrm{mol} / \mathrm{m}^{2}-\mathrm{s}\right]$ \\
\hline$i_{0}^{ \pm}$ & Exchange current density $\left[\mathrm{A} / \mathrm{m}^{2}\right]$ \\
\hline$\eta^{ \pm}$ & Overpotential [V] \\
\hline$c_{S S}^{ \pm}$ & Lithium concentration at solid particle surface $\left[\mathrm{mol} / \mathrm{m}^{3}\right]$ \\
\hline$\theta^{ \pm}$ & Normalized surface concentration $c_{s s}^{ \pm} / c_{s, \max }^{ \pm}[-]$ \\
\hline$I$ & Applied current $\left[\mathrm{A} / \mathrm{m}^{2}\right]$ \\
\hline$V$ & Terminal voltage [V] \\
\hline \multicolumn{2}{|r|}{ Electrochemical model parameters } \\
\hline$D_{s}^{ \pm}, D_{e}$ & Diffusivity of solid, electrolyte phase $\left[\mathrm{m}^{2} / \mathrm{s}\right]$ \\
\hline$t_{c}^{0}$ & Transference number $[-]$ \\
\hline$\varepsilon_{s}^{ \pm}, \varepsilon_{e}$ & Volume fraction of solid, electrolyte phase [-] \\
\hline$F$ & Faraday's constant $[\mathrm{C} / \mathrm{mol}]$ \\
\hline$\sigma^{ \pm}$ & Conductivity of solid $[1 / \Omega-\mathrm{m}]$ \\
\hline$\kappa$ & Conductivity of electrolyte $[1 / \Omega-\mathrm{m}]$ \\
\hline$R$ & Universal gas constant $[\mathrm{J} / \mathrm{mol}-\mathrm{K}]$ \\
\hline$T$ & Temperature $[\mathrm{K}]$ \\
\hline$f_{c / a}$ & Mean molar activity coefficient in electrolyte [-] \\
\hline$a^{ \pm}$ & Specific interfacial surface area $\left[\mathrm{m}^{2} / \mathrm{m}^{3}\right]$ \\
\hline$\alpha_{a}, \alpha_{c}$ & Anodic, cathodic charge transfer coefficient [-] \\
\hline$k^{ \pm}$ & Kinetic reaction rate $\left[\left(\mathrm{A} / \mathrm{m}^{2}\right)\left(\mathrm{mol}^{3} / \mathrm{mol}\right)^{(1+\alpha)}\right]$ \\
\hline$c_{s, \max }^{ \pm}$ & Maximum concentration of solid material $\left[\mathrm{mol} / \mathrm{m}^{3}\right]$ \\
\hline$U^{ \pm}$ & Open circuit potential of solid material [V] \\
\hline$R_{f}^{ \pm}$ & Solid-electrolyte interphase filme resistance $\left[\Omega-\mathrm{m}^{2}\right]$ \\
\hline$R_{s}^{ \pm}$ & Particle radius in solid phase $[\mathrm{m}]$ \\
\hline$L^{j}$ & Length of region $j \in\{-$, sep, +$\}$ \\
\hline \multicolumn{2}{|r|}{ Observer analysis / design parameters } \\
\hline$n_{L i, s}, n_{L i, e}$ & Total moles of lithium in solid, electrolyte phase [mol] \\
\hline$\lambda$ & Cathode estimation error system parameter \\
\hline$p^{ \pm}(r), p_{0}^{ \pm}$ & Cathode / anode observer gains \\
\hline$\hat{c}_{s s}^{+}$ & Estimated cathode surface concentration $\left[\mathrm{mol} / \mathrm{m}^{3}\right]$ \\
\hline$\check{c}_{s s}^{+}$ & Processed cathode surface concentration $\left[\mathrm{mol} / \mathrm{m}^{3}\right]$ \\
\hline$\gamma$ & Output inversion gain \\
\hline
\end{tabular}

[13] B. Saha, K. Goebel, S. Poll, and J. Christophersen, "Prognostics methods for battery health monitoring using a bayesian framework," IEEE Transactions on Instrumentation and Measurement, vol. 58, no. 2, pp. $291-296$, Feb 2009.

[14] S. Santhanagopalan and R. E. White, "Online estimation of the state of charge of a lithium ion cell," Journal of Power Sources, vol. 161, no. 2, pp. $1346-1355,2006$

[15] S. J. Moura, N. Chaturvedi, and M. Krstic, "Adaptive PDE Observer for Battery SOC/SOH Estimation via an Electrochemical Model," ASME Journal of Dynamic Systems, Measurement, and Control, vol. 136, no. 1, pp. 011015-011026, Oct 2014.

[16] S. Dey and B. Ayalew, "Nonlinear observer designs for state-of-charge estimation of lithium-ion batteries," in 2014 American Control Conference (ACC), June 2014, pp. 248-253.

[17] Y. Wang, H. Fang, Z. Sahinoglu, T. Wada, and S. Hara, "Adaptive estimation of the state of charge for lithium-ion batteries: Nonlinear geometric observer approach," IEEE Transactions on Control Systems Technology, vol. 23, no. 3, pp. 948-962, May 2015.

[18] E. Prada, D. Di Domenico, Y. Creff, J. Bernard, V. Sauvant-Moynot, and F. Huet, "Simplified electrochemical and thermal model of lifepo4graphite li-ion batteries for fast charge applications," Journal of The Electrochemical Society, vol. 159, no. 9, pp. A1508-A1519, 2012.

[19] S. K. Rahimian, S. Rayman, and R. E. White, "Extension of physicsbased single particle model for higher charge-discharge rates," Journal of Power Sources, vol. 224, no. 0, pp. 180 - 194, 2013. 
[20] P. Kemper and D. Kum, "Extended single particle model of li-ion batteries towards high current applications," in Vehicle Power and Propulsion Conference (VPPC), 2013 IEEE, Oct 2013, pp. 1-6.

[21] T. R. Tanim, C. D. Rahn, and C.-Y. Wang, "A temperature dependent, single particle, lithium ion cell model including electrolyte diffusion," ASME Journal of Dynamic Systems, Measurement, and Control, vol. 137, no. 1, p. 011005, 2015.

[22] X. Han, M. Ouyang, L. Lu, and J. Li, "Simplification of physics-based electrochemical model for lithium ion battery on electric vehicle. part i: Diffusion simplification and single particle model," Journal of Power Sources, vol. 278, no. 0, pp. $802-813,2015$.

[23] T. R. Tanim, C. D. Rahn, and C.-Y. Wang, "State of charge estimation of a lithium ion cell based on a temperature dependent and electrolyte enhanced single particle model," Energy, vol. 80, no. 0, pp. 731 - 739, 2015.

[24] X. Han, M. Ouyang, L. Lu, and J. Li, "Simplification of physics-based electrochemical model for lithium ion battery on electric vehicle. Part II: Pseudo-two-dimensional model simplification and state of charge estimation," Journal of Power Sources, vol. 278, no. 0, pp. $814-825$, 2015.

[25] R. Klein, N. A. Chaturvedi, J. Christensen, J. Ahmed, R. Findeisen, and A. Kojic, "Electrochemical Model Based Observer Design for a LithiumIon Battery," IEEE Transactions on Control Systems Technology, vol. 21, no. 2, pp. 289-301, March 2013.

[26] K. A. Smith, C. D. Rahn, and C.-Y. Wang, "Model-based electrochemical estimation and constraint management for pulse operation of lithium ion batteries," IEEE Transactions on Control Systems Technology, vol. 18, no. 3, pp. $654-663,2010$.

[27] M. Corno, N. Bhatt, S. Savaresi, and M. Verhaegen, "Electrochemical model-based state of charge estimation for li-ion cells," IEEE Transactions on Control Systems Technology, vol. 23, no. 1, pp. 117-127, Jan 2015.

[28] K. D. Stetzel, L. L. Aldrich, M. S. Trimboli, and G. L. Plett, "Electrochemical state and internal variables estimation using a reduced-order physics-based model of a lithium-ion cell and an extended kalman filter," Journal of Power Sources, vol. 278, no. 0, pp. 490 - 505, 2015.

[29] A. Bartlett, J. Marcicki, S. Onori, G. Rizzoni, X. G. Yang, and T. Miller, "Model-based state of charge estimation and observability analysis of a composite electrode lithium-ion battery," in 2013 IEEE 52nd Annual Conference on Decision and Control (CDC), Dec 2013, pp. 7791-7796.

[30] J. Newman. (2008) Fortran programs for the simulation of electrochemical systems. [Online]. Available: http://www.cchem. berkeley.edu/jsngrp/fortran.html

[31] S. Moura, J. Stein, and H. Fathy, "Battery-Health Conscious Power Management in Plug-In Hybrid Electric Vehicles via Electrochemical Modeling and Stochastic Control," IEEE Transactions on Control Systems Technology, vol. 21, no. 3, pp. 679-694, 2013.

[32] A. Smyshlyaev and M. Krstic, "Backstepping observers for a class of parabolic PDEs," Systems \& Control Letters, vol. 54, no. 7, pp. 613-625, 2005.

[33] A. P. Schmidt, M. Bitzer, A. W. Imre, and L. Guzzella, "Experimentdriven electrochemical modeling and systematic parameterization for a lithium-ion battery cell," Journal of Power Sources, vol. 195, no. 15, pp. $5071-5080,2010$.

[34] J. C. Forman, S. J. Moura, J. L. Stein, and H. K. Fathy, "Genetic identification and Fisher identifiability analysis of the Doyle-FullerNewman model from experimental cycling of a LiFePO4 cells," Journal of Power, vol. 210, pp. 263-275, 2012.

[35] A. Bartlett, J. Marcicki, S. Onori, G. Rizzoni, X. Yang, and T. Miller, "Electrochemical model-based state of charge and capacity estimation for a composite electrode lithium-ion battery," IEEE Transactions on Control Systems Technology, vol. PP, no. 99, pp. 1-1, 2015.

[36] P. Ioannou and J. Sun, Robust Adaptive Control. Prentice-Hall, 1996.

[37] M. Krstic and A. Smyshlyaev, Boundary Control of PDEs: A Course on Backstepping Designs. Philadelphia, PA: Society for Industrial and Applied Mathematics, 2008.

[38] R. Courant and D. Hilbert, Methods of Mathematical Physics. CUP Archive, 1966, vol. 1.

[39] X. Fan and M. Arcak, "Observer design for systems with multivariable monotone nonlinearities," Systems \& Control Letters, vol. 50, no. 4, pp. $319-330,2003$

[40] G. Phanomchoeng, R. Rajamani, and D. Piyabongkarn, "Nonlinear observer for bounded jacobian systems, with applications to automotive slip angle estimation," IEEE Transactions on Automatic Control, vol. 56, no. 5, pp. 1163-1170, May 2011.

[41] H. K. Khalil, Nonlinear Systems, 3rd ed. Prentice Hall, 2002.

[42] J. B. Siegel, X. Lin, A. G. Stefanopoulou, D. S. Hussey, D. L. Jacobson, and D. Gorsich, "Neutron imaging of lithium concentration in LFP Pouch cell battery," Journal of the Electrochemical Society, vol. 158, no. 5, pp. A523 - A529, 2011.

[43] A. Same, V. Battaglia, H.-Y. Tang, and J. W. Park, "In situ neutron radiography analysis of graphite/nca lithium-ion battery during overcharge," Journal of Applied Electrochemistry, vol. 42, no. 1, pp. 1-9, 2011.

[44] S. C. Nagpure, P. Mulligan, M. Canova, and L. R. Cao, "Neutron depth profiling of li-ion cell electrodes with a gas-controlled environment," Journal of Power Sources, vol. 248, pp. 489 - 497, 2014.

[45] C. D. Rahn and C.-Y. Wang, Battery Systems Engineering. John Wiley \& Sons, 2012

[46] M. Canova, K. Pan, and G. Fan, "A Comparison of Model Order Reduction Techniques for Electrochemical Characterization of LithiumIon Batteries," in 54th IEEE Conference on Decision and Control, Osaka, Japan, 2015.

[47] J. Strikwerda, Finite difference schemes and partial differential equations. Society for Industrial and Applied Mathematics, 2007.

[48] H. E. Perez and S. J. Moura, "Sensitivity-based Interval PDE Observer for Battery SOC Estimation," in 2015 American Control Conference, Chicago, IL USA, 2015, pp. 323-328, [Best Student Paper Finalist]. 